\title{
The Orbit Rigidity Matrix of a Symmetric Framework
}

\author{
Bernd Schulze • Walter Whiteley
}

Received: 4 June 2010 / Revised: 27 November 2010 / Accepted: 4 December 2010 /

Published online: 29 December 2010

(C) Springer Science+Business Media, LLC 2010

\begin{abstract}
A number of recent papers have studied when symmetry causes frameworks on a graph to become infinitesimally flexible, or stressed, and when it has no impact. A number of other recent papers have studied special classes of frameworks on generically rigid graphs which are finite mechanisms. Here we introduce a new tool, the orbit matrix, which connects these two areas and provides a matrix representation for fully symmetric infinitesimal flexes, and fully symmetric stresses of symmetric frameworks. The orbit matrix is a true analog of the standard rigidity matrix for general frameworks, and its analysis gives important insights into questions about the flexibility and rigidity of classes of symmetric frameworks, in all dimensions.

With this narrower focus on fully symmetric infinitesimal motions, comes the power to predict symmetry-preserving finite mechanisms_-giving a simplified analysis which covers a wide range of the known mechanisms, and generalizes the classes of known mechanisms. This initial exploration of the properties of the orbit matrix also opens up a number of new questions and possible extensions of the previous results, including transfer of symmetry based results from Euclidean space to spherical, hyperbolic, and some other metrics with shared symmetry groups and underlying projective geometry.
\end{abstract}

B. Schulze was supported by the DFG Research Unit 565 'Polyhedral Surfaces'. W. Whiteley was supported by a grant from NSERC (Canada).

B. Schulze $(\varangle)$

Institute of Mathematics, MA 6-2 Technische Universität Berlin, Strasse des 17. Juni 136, 10623

Berlin, Germany

e-mail: bschulze@math.tu-berlin.de

W. Whiteley

Department of Mathematics and Statistics, York University, 4700 Keele Street, Toronto, ON

M3J1P3, Canada

e-mail: whiteley@ mathstat.yorku.ca 
Keywords Bar and joint frameworks · Symmetry · Orbits · Infinitesimal motion · Finite motion $\cdot$ Mechanism

\section{Introduction}

Over the last decade, a substantial theory on the interactions of symmetry and rigidity has been developed [14, 18, 21, 24-26, 32, 34, 35]. This includes descriptions of when symmetry changes generically rigid graphs into infinitesimally flexible frameworks, and when symmetry does not modify the behavior. These analyses have used tools of representation theory to analyze the stresses and motions of the symmetric realizations of a graph. Some extensions have gone further to describe situations when the symmetry switches a graph into configurations with symmetry-preserving finite flexes [20,32]. These predictions of finite symmetric flexes turn out to focus on frameworks with fully symmetric infinitesimal flexes, and with fully symmetric self-stresses [33].

There is a companion, extensive literature on flexible frameworks built on generically rigid frameworks, starting with Bricard's flexible octahedra [8, 39], running though linkages such as Bottema's mechanism [7] and other finitely flexible frameworks [12] and Connelly's flexible sphere [10] to recent work on flexible crosspolytopes in 4-space [40]. Some of this work has looked at creating analog examples of finite mechanisms in other metrics such as the spherical and hyperbolic space [1]. In general, it is a difficult task to decide when a specific infinitesimal flex of a framework on a generically rigid graph extends to a finite flex. However, on careful examination, many of these known examples have symmetries and infinitesimal flexes which preserve this symmetry [33, 42]. It is natural to seek tools and connections that can simplify the creation and generation of such examples of finite mechanisms (linkages).

In [33], the block of the block-decomposed rigidity matrix which corresponds to the trivial irreducible representation of the point group of the framework was used to study the spaces of fully symmetric motions and fully symmetric self-stresses of the framework. This analysis gave some initial results predicting finite flexes which remain fully symmetric throughout their path. However, actual generation of this block in the decomposition required substantial machinery from representation theory, and the entries in the matrix were not transparent.

In this paper we present the orbit matrix for a symmetric framework as an original, simplifying tool for detecting this whole package of fully symmetric infinitesimal flexes, fully symmetric self-stresses, and predicting finite flexes for configurations which are generic within the symmetry. In our proofs, we will actually show that the orbit matrix is equivalent to the matrix studied in [33], but the construction is transparent, and the entries in the matrix are explicitly derived. For a framework with a nontrivial point group, this orbit matrix has a set of columns for each orbit of vertices under the group action, and one row for each orbit of edges under the group action. We will give a detailed construction for this matrix in Sect. 5, and show that the kernel is precisely the fully symmetric infinitesimal motions (Sects. 4, 6) and the row dependencies are exactly the fully symmetric self-stresses (Sects. 4, 8). This orbit matrix provides a powerful tool for investigating many aspects of the behavior of fully symmetric frameworks on the graph. 
From the counts of the columns $c$, the rows $r$, and the dimension of the fully symmetric trivial infinitesimal motions $m$, we can give some immediate sufficient conditions for the presence of fully symmetric infinitesimal flexes (see Sect. 7). Moreover, at configurations in which the representative vertices for the orbits are chosen 'generically', the presence of a fully symmetric infinitesimal flex is a guarantee of a symmetry-preserving finite flex. With these tools for counting under symmetry, we have direct predictions of flexible frameworks which capture many of the classical examples, including two types of flexible octahedra, the Bottema mechanism, and the flexible cross-polytopes. A striking example of a general class covered by this analysis is the following:

Theorem 7.5 Given a graph which is generically isostatic in 3-space, and a framework on the graph realized in 3-space as generically as possible with 2-fold symmetry with no vertices or edges fixed by the rotation, the framework has a finite flex preserving the symmetry.

Because this orbit matrix is a powerful symmetry adapted analog of the standard rigidity matrix, many of the questions from standard rigidity have extensions for fully symmetric stresses and motions. Some of these questions and possible extensions are presented in Sect. 9, along with brief discussions of the potential for symmetry adapted extensions of the techniques and results. As one example, it is natural to seek analogs of Laman's Theorem to characterize necessary and sufficient conditions for the orbit matrix of a graph and symmetry group to be independent with maximal rank. As a second example, because key portions of the point group symmetries and the corresponding counting for this orbit matrix can be transferred to other metrics (such as the spherical, hyperbolic and Minkowski spaces), the methods developed here provide a uniform construction of mechanisms such as the Bricard octahedron, the flexible cross-polytope, and the Bottema mechanism and its generalizations across multiple metrics.

While the analysis of the fully symmetric rigidity properties of a framework with a non-trivial point group can be simplified significantly by studying its orbit rigidity matrix instead of the submatrix block in its block-decomposed rigidity matrix which corresponds to the trivial irreducible representation of the point group (see [34]), this can in general not be done for the other submatrix blocks which correspond to the non-trivial irreducible representations of the group.

However, for each non-trivial irreducible representation, we may detect infinitesimal flexes of the framework which exhibit the symmetry of this representation by either constructing the block-decomposed rigidity matrix of the framework explicitly using techniques from group representation theory, and computing the rank of the submatrix block corresponding to this representation, or, in some cases, by simply determining the size of this submatrix block using techniques from character theory (see $[18,34]$, for example). Note that if we are given a framework which counts to be isostatic, and we detect a fully-symmetric self-stress in this framework (using the methods presented in this paper, for example), then we may conclude that this framework must have an infinitesimal flex which exhibits the symmetry of some non-trivial irreducible representation of the point group. However, to determine the exact symmetry of this flex, one needs to apply the methods in [18, 34]. 
Analogously to the fully-symmetric case, it is shown in [33] that an infinitesimal flex which is symmetric with respect to a non-trivial irreducible representation also extends to a finite mechanism, provided that the configuration is a 'regular' point for an appropriately defined algebraic variety. So, in particular, this allows us to detect finite mechanisms in symmetric frameworks that preserve a subgroup of the point group of the original framework, but break the other symmetries. However, to determine whether a given configuration is in fact a regular point for the algebraic variety associated with a non-trivial irreducible representation is not as straightforward as in the fully-symmetric case (see [33] for further details).

The orbit matrix, as a tool for analyzing frameworks subject to a group action, has analogs in current work on periodic frameworks [6, 27, 28]. A recent paper has combined the work on periodic frameworks and this work on symmetric frameworks to create related orbit matrices from which predictions of finite motions can also be made [29].

As a final comment, key results on the global rigidity of generic frameworks depend on self-stresses and the equivalence of finite flexes and infinitesimal flexes for generic frameworks. We now have fully-symmetric versions of these tools, and can extract some analogs of the global rigidity results for symmetry-generic frameworks, both as conditions under which they are globally rigid within the class of fully-symmetric frameworks, and when they are globally rigid within the class of all frameworks. Still there are additional conjectures and new results to be explored in this area.

We hope that this paper serves as an invitation for the reader to join in the further explorations of these many levels of interactions of symmetry, rigidity, and flexibility.

\section{Rigidity Theoretic Definitions and Preliminaries}

All graphs considered in this paper are finite graphs without loops or multiple edges. The vertex set of a graph $G$ is denoted by $V(G)$ and the edge set of $G$ is denoted by $E(G)$.

A framework in $\mathbb{R}^{d}$ is a pair $(G, p)$, where $G$ is a graph and $p: V(G) \rightarrow \mathbb{R}^{d}$ is a map such that $p(u) \neq p(v)$ for all $\{u, v\} \in E(G)$. We also say that $(G, p)$ is a $d$ dimensional realization of the underlying graph $G[19,49]$. For $v \in V(G)$, we say that $p(v)$ is the joint of $(G, p)$ corresponding to $v$, and for $e \in E(G)$, we say that $p(e)$ is the bar of $(G, p)$ corresponding to $e$.

For a framework $(G, p)$ whose underlying graph $G$ has the vertex set $V(G)=$ $\{1, \ldots, n\}$, we will frequently denote the vector $p(i)$ by $p_{i}$ for each $i$. The $k$ th component of a vector $x$ is denoted by $(x)_{k}$. It is often useful to identify $p$ with a vector in $\mathbb{R}^{d n}$ by using the order on $V(G)$. In this case we also refer to $p$ as a configuration of $n$ points in $\mathbb{R}^{d}$. Throughout this paper, we do not differentiate between an abstract vector and its coordinate column vector relative to the canonical basis.

A framework $(G, p)$ in $\mathbb{R}^{d}$ with $V(G)=\{1, \ldots, n\}$ is flexible if there exists a continuous path, called a finite flex or mechanism, $p(t):[0,1] \rightarrow \mathbb{R}^{d n}$ such that

(i) $p(0)=p$;

(ii) $\left\|p(t)_{i}-p(t)_{j}\right\|=\left\|p_{i}-p_{j}\right\|$ for all $0 \leq t \leq 1$ and all $\{i, j\} \in E(G)$; 
(iii) $\left\|p(t)_{k}-p(t)_{l}\right\| \neq\left\|p_{k}-p_{l}\right\|$ for all $0<t \leq 1$ and some pair $\{k, l\}$ of vertices of $G$.

Otherwise $(G, p)$ is said to be rigid. For some alternate equivalent definitions of a rigid and flexible framework see [2, 30], for example.

An infinitesimal motion of a framework $(G, p)$ in $\mathbb{R}^{d}$ with $V(G)=\{1, \ldots, n\}$ is a function $u: V(G) \rightarrow \mathbb{R}^{d}$ such that

$$
\left(p_{i}-p_{j}\right)^{T}\left(u_{i}-u_{j}\right)=0 \text { for all }\{i, j\} \in E(G),
$$

where $u_{i}$ denotes the column vector $u(i)$ for each $i$.

An infinitesimal motion $u$ of $(G, p)$ is an infinitesimal rigid motion (or trivial infinitesimal motion) if there exists a skew-symmetric matrix $S$ (a rotation) and a vector $t$ (a translation) such that $u(v)=S p(v)+t$ for all $v \in V(G)$. Otherwise $u$ is an infinitesimal flex (or non-trivial infinitesimal motion) of $(G, p)$.

$(G, p)$ is infinitesimally rigid if every infinitesimal motion of $(G, p)$ is an infinitesimal rigid motion. Otherwise $(G, p)$ is said to be infinitesimally flexible $[19,49]$.

The rigidity matrix of $(G, p)$ is the $|E(G)| \times d n$ matrix

$$
\begin{aligned}
& \mathbf{R}(G, p)
\end{aligned}
$$

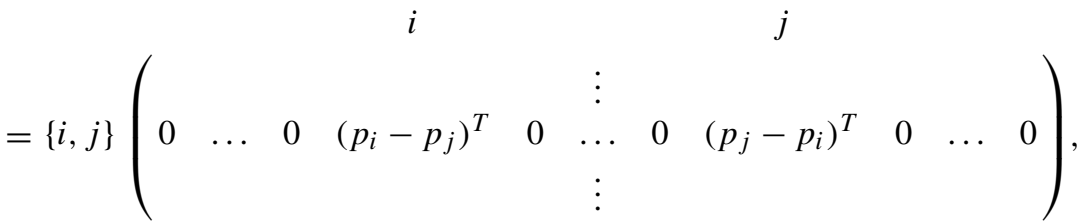

that is, for each edge $\{i, j\} \in E(G), \mathbf{R}(G, p)$ has the row with $\left(p_{i}-p_{j}\right)_{1}, \ldots,\left(p_{i}-\right.$ $\left.p_{j}\right)_{d}$ in the columns $d(i-1)+1, \ldots, d i,\left(p_{j}-p_{i}\right)_{1}, \ldots,\left(p_{j}-p_{i}\right)_{d}$ in the columns $d(j-1)+1, \ldots, d j$, and 0 elsewhere $[19,49]$.

Note that if we identify an infinitesimal motion $u$ of $(G, p)$ with a column vector in $\mathbb{R}^{d n}$ (by using the order on $V(G)$ ), then the equations in (1) can be written as $\mathbf{R}(G, p) u=0$. So, the kernel of the rigidity matrix $\mathbf{R}(G, p)$ is the space of all infinitesimal motions of $(G, p)$. It is well known that a framework $(G, p)$ in $\mathbb{R}^{d}$ is infinitesimally rigid if and only if either the rank of its associated rigidity matrix $\mathbf{R}(G, p)$ is precisely $d n-\left(\begin{array}{c}d+1 \\ 2\end{array}\right)$, or $G$ is a complete graph $K_{n}$ and the points $p_{i}$, $i=1, \ldots, n$, are affinely independent [2].

While an infinitesimally rigid framework is always rigid, the converse does not hold in general. Asimov and Roth, however, showed that for 'generic' configurations, infinitesimal rigidity and rigidity are in fact equivalent [2].

A self-stress of a framework $(G, p)$ with $V(G)=\{1, \ldots, n\}$ is a function $\omega$ : $E(G) \rightarrow \mathbb{R}$ such that at each joint $p_{i}$ of $(G, p)$ we have

$$
\sum_{j:\{i, j\} \in E(G)} \omega_{i j}\left(p_{i}-p_{j}\right)=0,
$$

where $\omega_{i j}$ denotes $\omega(\{i, j\})$ for all $\{i, j\} \in E(G)$. Note that if we identify a selfstress $\omega$ with a column vector in $\mathbb{R}^{|E(G)|}$ (by using the order on $E(G)$ ), then we have 
$\omega^{T} \mathbf{R}(G, p)=0$. In structural engineering, the self-stresses are also called equilibrium stresses as they record tensions and compressions in the bars balancing at each vertex.

If $(G, p)$ has a non-zero self-stress, then $(G, p)$ is said to be dependent (since in this case there exists a linear dependency among the row vectors of $\mathbf{R}(G, p)$ ). Otherwise, $(G, p)$ is said to be independent. A framework which is both independent and infinitesimally rigid is called isostatic [16, 46, 49].

\section{Symmetry in Frameworks}

Let $G$ be a graph with $V(G)=\{1, \ldots, n\}$, and let $\operatorname{Aut}(G)$ denote the automorphism group of $G$. A symmetry operation of a framework $(G, p)$ in $\mathbb{R}^{d}$ is an isometry $x$ of $\mathbb{R}^{d}$ such that for some $\alpha \in \operatorname{Aut}(G)$, we have $x\left(p_{i}\right)=p_{\alpha(i)}$ for all $i \in V(G)$ [22, $32,34,35]$.

The set of all symmetry operations of a framework $(G, p)$ forms a group under composition, called the point group of $(G, p)[4,22,32,35]$. Since translating a framework does not change its rigidity properties, we may assume wlog that the point group of any framework in this paper is a symmetry group, i.e., a subgroup of the orthogonal group $O\left(\mathbb{R}^{d}\right)[32,34,35]$.

We use the Schoenflies notation for the symmetry operations and symmetry groups considered in this paper, as this is one of the standard notations in the literature about symmetric structures (see [4, 14, 18, 20, 22, 24, 25, 32, 34, 35], for example). In this notation, the identity transformation is denoted by $I d$, a rotation about a $(d-2)$ dimensional subspace of $\mathbb{R}^{d}$ by an angle of $\frac{2 \pi}{m}$ is denoted by $C_{m}$, and a reflection in a $(d-1)$-dimensional subspace of $\mathbb{R}^{d}$ is denoted by $s$.

While the general results of this paper apply to all symmetry groups, we will only analyze examples with four types of groups. In the Schoenflies notation, they are denoted by $\mathcal{C}_{s}, \mathcal{C}_{m}, \mathcal{C}_{m v}$, and $\mathcal{C}_{m h}$. For any dimension $d, \mathcal{C}_{s}$ is a symmetry group consisting of the identity $I d$ and a single reflection $s$, and $\mathcal{C}_{m}$ is a cyclic group generated by a rotation $C_{m}$. The only other possible type of symmetry group in dimension 2 is the group $\mathcal{C}_{m v}$ which is a dihedral group generated by a pair $\left\{C_{m}, s\right\}$. In dimension $d>2, \mathcal{C}_{m v}$ denotes any symmetry group that is generated by a rotation $C_{m}$ and a reflection $s$ whose corresponding mirror contains the rotational axis of $C_{m}$, whereas a symmetry group $\mathcal{C}_{m h}$ is generated by a rotation $C_{m}$ and the reflection $s$ whose corresponding mirror is perpendicular to the $C_{m}$-axis. For further information about the Schoenflies notation we refer the reader to $[4,22,32]$.

Given a symmetry group $S$ in dimension $d$ and a graph $G$, we let $\mathcal{R}_{(G, S)}$ denote the set of all $d$-dimensional realizations of $G$ whose point group is either equal to $S$ or contains $S$ as a subgroup [32,34,35]. In other words, the set $\mathcal{R}_{(G, S)}$ consists of all realizations $(G, p)$ of $G$ for which there exists a map $\Phi: S \rightarrow \operatorname{Aut}(G)$ so that

$$
x\left(p_{i}\right)=p_{\Phi(x)(i)} \quad \text { for all } i \in V(G) \text { and all } x \in S .
$$

A framework $(G, p) \in \mathcal{R}_{(G, S)}$ satisfying the equations in (2) for the map $\Phi: S \rightarrow$ $\operatorname{Aut}(G)$ is said to be of type $\Phi$, and the set of all realizations in $\mathcal{R}_{(G, S)}$ which are of type $\Phi$ is denoted by $\mathcal{R}_{(G, S, \Phi)}$ (see again [32, 34, 35] and Fig. 1). 


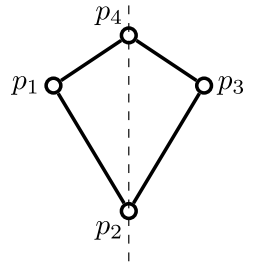

(a)

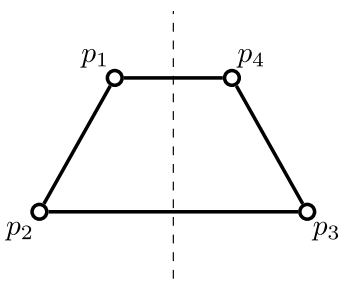

(b)

Fig. 1 2-dimensional realizations of $K_{2,2}$ in $\mathcal{R}_{\left(K_{2,2}, \mathcal{C}_{s}\right)}$ of different types: the framework in (a) is of type $\Phi_{a}$, where $\Phi_{a}: \mathcal{C}_{s} \rightarrow \operatorname{Aut}\left(K_{2,2}\right)$ is the homomorphism defined by $\Phi_{a}(s)=(13)(2)(4)$ and the framework in (b) is of type $\Phi_{b}$, where $\Phi_{b}: \mathcal{C}_{s} \rightarrow \operatorname{Aut}\left(K_{2,2}\right)$ is the homomorphism defined by $\Phi_{b}(s)=(14)(23)$

It is shown in $[32,35]$ that if the map $p$ of a framework $(G, p) \in \mathcal{R}_{(G, S)}$ is injective, then $(G, p)$ is of a unique type $\Phi$ and $\Phi$ is necessarily also a homomorphism. For simplicity, we therefore assume that the map $p$ of any framework $(G, p)$ considered in this paper is injective (i.e., $p_{i} \neq p_{j}$ if $i \neq j$ ). In particular, this allows us (with a slight abuse of notation) to use the terms $p_{x(i)}$ and $p_{\Phi(x)(i)}$ interchangeably, where $i \in V(G)$ and $x \in S$. In general, if the type $\Phi$ is clear from the context, we often simply write $x(i)$ instead of $\Phi(x)(i)$.

Let $(G, p) \in \mathcal{R}_{(G, S, \Phi)}$ and let $x$ be a symmetry operation in $S$. Then the joint $p_{i}$ of $(G, p)$ is said to be fixed by $x$ if $x\left(p_{i}\right)=p_{i}$ (or equivalently, $x(i)=i$ ).

Let the symmetry element corresponding to $x$ be the linear subspace $F_{x}$ of $\mathbb{R}^{d}$ which consists of all points $a \in \mathbb{R}^{d}$ with $x(a)=a$. Then the joint $p_{i}$ of any framework $(G, p)$ in $\mathcal{R}_{(G, S, \Phi)}$ must lie in the linear subspace

$$
U\left(p_{i}\right)=\bigcap_{x \in S: x\left(p_{i}\right)=p_{i}} F_{x}
$$

Note that $U\left(p_{i}\right) \neq \emptyset$, because the origin is fixed by every symmetry operation in the symmetry group $S$.

Example 3.1 The joint $p_{1}$ of the framework $\left(K_{2,2}, p\right) \in \mathcal{R}_{\left(K_{2,2}, \mathcal{C}_{s}, \Phi_{a}\right)}$ depicted in Fig. 1(a) is fixed by the identity $I d \in \mathcal{C}_{s}$, but not by the reflection $s \in \mathcal{C}_{s}$, so that $U\left(p_{1}\right)=F_{I d}=\mathbb{R}^{d}$. The joint $p_{2}$ of $\left(K_{2,2}, p\right)$, however, is fixed by both the identity $I d$ and the reflection $s$ in $\mathcal{C}_{s}$, so that $U\left(p_{2}\right)=F_{I d} \cap F_{S}=F_{s}$. In other words, $U\left(p_{2}\right)$ is the mirror line corresponding to $s$.

Note that if we choose a set of representatives $\mathcal{O}_{V(G)}=\{1, \ldots, k\}$ for the orbits $S(i)=\{\Phi(x)(i) \mid x \in S\}$ of vertices of $G$, then the positions of all joints of $(G, p) \in \mathcal{R}_{(G, S, \Phi)}$ are uniquely determined by the positions of the joints $p_{1}, \ldots, p_{k}$ and the symmetry constraints imposed by $S$ and $\Phi$. Thus, any framework in $\mathcal{R}_{(G, S, \Phi)}$ may be constructed by first choosing positions $p_{i} \in U\left(p_{i}\right)$ for each $i=1, \ldots, k$, and then letting $S$ and $\Phi$ determine the positions of the remaining joints. In particular, by placing the vertices of $\mathcal{O}_{V(G)}$ into 'generic' positions within their associated subspaces $U\left(p_{i}\right)$ we obtain an $(S, \Phi)$-generic realization of $G$ (i.e., a realization of $G$ 
that is as 'generic' as possible within the set $\left.\mathcal{R}_{(G, S, \Phi)}\right)$ in this way. For a precise definition of $(S, \Phi)$-generic, and further information about $(S, \Phi)$-generic frameworks, we refer the reader to $[32,33,35]$.

\section{Fully Symmetric Motions and Self-Stresses}

An infinitesimal motion $u$ of a framework $(G, p) \in \mathcal{R}_{(G, S, \Phi)}$ is fully $(S, \Phi)$ symmetric if

$$
x\left(u_{i}\right)=u_{\Phi(x)(i)} \quad \text { for all } i \in V(G) \quad \text { and all } x \in S,
$$

i.e., if $u$ is unchanged under all symmetry operations in $S$ (see also Fig. 2(a) and (b)).

Note that it follows immediately from (3) that if $u$ is a fully $(S, \Phi)$-symmetric infinitesimal motion of $(G, p)$, then $u_{i}$ is an element of $U\left(p_{i}\right)$ for each $i$. Moreover, $u$ is uniquely determined by the velocity vectors $u_{1}, \ldots, u_{k}$ whenever $\mathcal{O}_{V(G)}=\{1, \ldots, k\}$ is a set of representatives for the vertex orbits $S(i)=\{\Phi(x)(i) \mid x \in S\}$ of $G$.

Example 4.1 Consider the framework shown in Fig. 2(a). With $p_{1}^{T}=(a, b), p_{2}^{T}=$ $(0, c), p_{3}^{T}=(-a, b)$, and $p_{4}^{T}=(0, d)$ the rigidity matrix of $\left(K_{2,2}, p\right)$ has the form

$$
\begin{aligned}
& \{1,2\} \\
& \{1,4\} \\
& s(\{1,2\}) \\
& s(\{1,4\})
\end{aligned}\left(\begin{array}{cccc}
(a, b-c) & (-a, c-b) & 00 & 4 \\
(a, b-d) & 00 & 00 & (-a, d-b) \\
00 & (a, c-b) & (-a, b-c) & 00 \\
00 & 00 & (-a, b-d) & (a, d-b)
\end{array}\right) .
$$

This matrix has rank 4 , and hence leaves a space of $8-4=4$ infinitesimal motions. Thus, there exists a 1-dimensional space of infinitesimal flexes of $\left(K_{2,2}, p\right)$ spanned by $u=\left(-100 \frac{a}{c-b} 100 \frac{a}{d-b}\right)^{T}$. This infinitesimal flex is clearly fully $\left(\mathcal{C}_{s}, \Phi_{a}\right)$ symmetric.

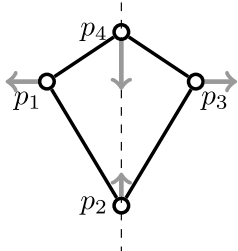

(a)

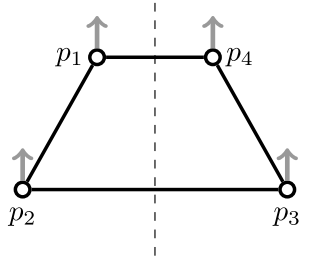

(b)

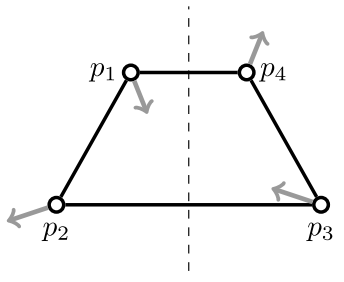

(c)

Fig. 2 Infinitesimal motions of frameworks in the plane: (a) a fully $\left(\mathcal{C}_{s}, \Phi_{a}\right)$-symmetric non-trivial infinitesimal motion of $\left(K_{2,2}, p\right) \in \mathcal{R}_{\left(K_{2,2}, \mathcal{C}_{s}, \Phi_{a}\right)}$; (b) a fully $\left(\mathcal{C}_{s}, \Phi_{b}\right)$-symmetric trivial infinitesimal motion of $\left(K_{2,2}, p\right) \in \mathcal{R}_{\left(K_{2,2}, \mathcal{C}_{s}, \Phi_{b}\right)}$; (c) a non-trivial infinitesimal motion of $\left(K_{2,2}, p\right) \in \mathcal{R}_{\left(K_{2,2}, \mathcal{C}_{s}, \Phi_{b}\right)}$ which is not fully $\left(\mathcal{C}_{S}, \Phi_{b}\right)$-symmetric 
Example 4.2 The rigidity matrix of the framework $\left(K_{2,2}, p\right)$ shown in Fig. 2(b), (c) with $p_{1}^{T}=(a, b), p_{2}^{T}=(c, d), p_{3}^{T}=(-c, d)$, and $p_{4}^{T}=(-a, b)$ has the form

\begin{tabular}{|c|c|c|c|c|}
\hline & 1 & 2 & $3=s(2)$ & $4=s(1)$ \\
\hline$\{1,2\}$ & $((a-c, b-d)$ & $(c-a, d-b)$ & 00 & 00 \\
\hline$\{1,4\}$ & $(2 a, 0)$ & 00 & 00 & $(-2 a, 0)$ \\
\hline$\{2,3\}$ & 00 & $(2 c, 0)$ & $(-2 c, 0)$ & 00 \\
\hline$s(\{1,2\})$ & 00 & 00 & $(a-c, d-b)$ & $(c-a, b-d)$ \\
\hline
\end{tabular}

This matrix has again rank 4 , and leaves a space of $8-4=4$ infinitesimal motions. The 1-dimensional space of infinitesimal flexes of $\left(K_{2,2}, p\right)$ is spanned by $u=\left(1-1-1 \frac{2(c-a)+b-d}{d-b}-1-\frac{2(c-a)+b-d}{d-b} 11\right)^{T}$. This infinitesimal flex is clearly not fully $\left(\mathcal{C}_{s}, \Phi_{b}\right)$-symmetric.

A self-stress $\omega$ of a framework $(G, p) \in \mathcal{R}_{(G, S, \Phi)}$ is fully $(S, \Phi)$-symmetric if $(\omega)_{e}=(\omega)_{f}$ whenever $e$ and $f$ belong to the same orbit $S(e)=\{\Phi(x)(e) \mid x \in S\}$ of edges of $G$ (see also Fig. 3(a)).

Note that a fully $(S, \Phi)$-symmetric self-stress is clearly uniquely determined by the components $(\omega)_{1}, \ldots,(\omega)_{r}$, whenever $\mathcal{O}_{E(G)}=\left\{e_{1}, \ldots, e_{r}\right\}$ is a set of representatives for the edge orbits $S(e)=\{\Phi(x)(e) \mid x \in S\}$ of $G$.

It is shown in $[25,34]$ that the rigidity matrix of a framework $(G, p) \in \mathcal{R}_{(G, S, \Phi)}$ can be transformed into a block-diagonalized form using techniques from group representation theory. In this block-diagonalization of $\mathbf{R}(G, p)$, the submatrix block $\tilde{\mathbf{R}}_{1}(G, p)$ that corresponds to the trivial irreducible representation of $S$ describes the relationship between external displacement vectors on the joints and resulting internal distortion vectors in the bars of $(G, p)$ that are fully $(S, \Phi)$-symmetric. So, the submatrix block $\tilde{\mathbf{R}}_{1}(G, p)$ comprises all the information regarding the fully $(S, \Phi)$ symmetric infinitesimal rigidity properties of $(G, p)$. The orbit rigidity matrix of $(G, p)$ which we will introduce in the next section will have the same properties as the submatrix block $\tilde{\mathbf{R}}_{1}(G, p)$; however, we will see that the orbit rigidity matrix allows a significantly simplified fully $(S, \Phi)$-symmetric infinitesimal rigidity analysis of $(G, p)$, since it can be set up directly without finding the block-diagonalization of $\mathbf{R}(G, p)$ or using other group representation techniques.

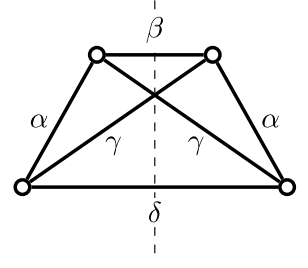

(a)

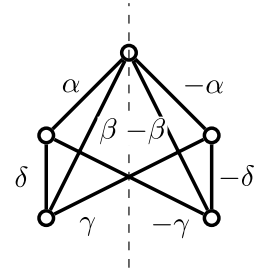

(b)

Fig. 3 Self-stressed frameworks in the plane: (a) a fully $\left(\mathcal{C}_{S}, \Phi\right)$-symmetric self-stress of

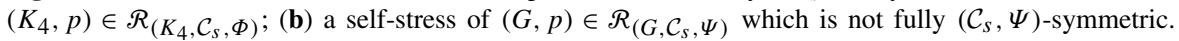
The types $\Phi$ and $\Psi$ are uniquely determined by the injective realizations [32, 35] 


\section{The Orbit Rigidity Matrix}

To make the general definition of the orbit rigidity matrix more transparent, we first consider a few simple examples.

Example 5.1 Consider the 2-dimensional framework $\left(K_{2,2}, p\right) \in \mathcal{R}_{\left(K_{2,2}, \mathcal{C}_{2}, \Phi\right)}$ depicted in Fig. 4, where $\Phi: \mathcal{C}_{2} \rightarrow \operatorname{Aut}\left(K_{2,2}\right)$ is the homomorphism defined by $\Phi\left(C_{2}\right)=(13)(24)$.

If we denote $p_{1}^{T}=(a, b), p_{2}^{T}=(c, d), p_{3}^{T}=(-a,-b)$, and $p_{4}^{T}=(-c,-d)$, then the rigidity matrix of $\left(K_{2,2}, p\right)$ is

\begin{tabular}{lcccc}
\multicolumn{1}{c}{1} & 2 & $3=C_{2}(1)$ & $4=C_{2}(2)$ \\
$\{1,2\}$ & $(a-c, b-d)$ & $(c-a, d-b)$ & 00 & 00 \\
$\{1,4\}$ & $(a+c, b+d)$ & 00 & 00 & $(-a-c,-b-d)$ \\
$C_{2}\{1,2\}$ & $C_{2}\{1,4\}$
\end{tabular}$\left(\begin{array}{cccc}00 & 0 & (c-a, d-b) & (a-c, b-d) \\
00 & (a+c, b+d) & (-a-c,-b-d) & 00\end{array}\right)$.

This matrix has rank 4 , and hence leaves a space of $8-4=4$ infinitesimal motions. Thus, there exists a 1-dimensional space of infinitesimal flexes of $\left(K_{2,2}, p\right)$ spanned by $u=\left(\begin{array}{lllllll}-1 & 0 & x & y & 1 & 0 & -x-y\end{array}\right)^{T}$, where $x=\frac{c d-a b}{a d-b c}$ and $y=-\frac{c^{2}-a^{2}}{a d-b c}$. This infinitesimal flex is clearly fully $\left(\mathcal{C}_{2}, \Phi\right)$-symmetric.

Note that if we are only interested in infinitesimal motions and self-stresses of $\left(K_{2,2}, p\right)$ that are fully $\left(\mathcal{C}_{2}, \Phi\right)$-symmetric, then it suffices to focus on the first two rows of $\mathbf{R}\left(K_{2,2}, p\right)$ (i.e., the rows corresponding to the representatives $\{1,2\}$ and $\{1,4\}$ for the edge orbits $S(e)=\left\{\Phi(x)(e) \mid x \in \mathcal{C}_{2}\right\}$ of $\left.K_{2,2}\right)$. The other two rows are redundant in this fully symmetric context. So, the orbit rigidity matrix for $\left(K_{2,2}, p\right)$ will have two rows, one for each representative of the edge orbits under the action of $\mathcal{C}_{2}$. Further, the orbit rigidity matrix will have only four columns, because each of the joints $p_{1}$ and $p_{2}$ has two degrees of freedom, and the displacement vectors at the joints $p_{3}=C_{2}\left(p_{1}\right)$ and $p_{4}=C_{2}\left(p_{2}\right)$ are uniquely determined by the displacement vectors at the joints $p_{1}$ and $p_{2}$ and the symmetry constraints given by $\mathcal{C}_{2}$ and $\Phi$. We write the orbit rigidity matrix of $\left(K_{2,2}, p\right)$ as follows:

12

$$
\begin{gathered}
\begin{array}{l}
\{1,2\} \\
\{1,4\}
\end{array}\left(\begin{array}{cc}
\left(p_{1}-p_{2}\right)^{T} & \left(p_{2}-p_{1}\right)^{T} \\
\left(p_{1}-C_{2}\left(p_{2}\right)\right)^{T} & \left(p_{2}-C_{2}^{-1}\left(p_{1}\right)\right)^{T}
\end{array}\right) \\
1 \\
=\left(\begin{array}{cc}
(a-c, b-d) & (c-a, d-b) \\
(a+c, b+d) & (c+a, d+b)
\end{array}\right) .
\end{gathered}
$$

Fig. 4 A framework $\left(K_{2,2}, p\right) \in \mathcal{R}_{\left(K_{2,2}, \mathcal{C}_{2}, \Phi\right)}$

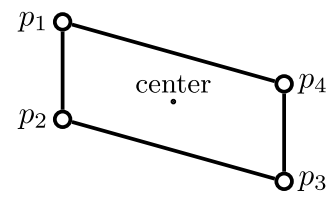


Example 5.2 The orbit rigidity matrix for the framework $\left(K_{2,2}, p\right) \in \mathcal{R}_{\left(K_{2,2}, \mathcal{C}_{s}, \Phi_{a}\right)}$ in Example 4.1 (Fig. 2(a)) has again two rows, since $K_{2,2}$ has two edge orbits (each of size 2) under the action of $\mathcal{C}_{s}$. The vertex orbits are represented by the vertices 1,2 and 4 , for example. Clearly, the joint $p_{1}$ has two degrees of freedom, which gives rise to two columns in the orbit matrix. The joints $p_{2}$ and $p_{4}$, however, are fixed by the reflection $s$ in $\mathcal{C}_{s}$, so that any fully $\left(\mathcal{C}_{s}, \Phi_{a}\right)$-symmetric displacement vectors at $p_{2}$ and $p_{4}$ must lie on the mirror corresponding to $s$ (i.e., on the $y$-axis). Thus, the orbit rigidity matrix of $\left(K_{2,2}, p\right)$ has only one column for each of the joints $p_{2}$ and $p_{4}$ :

$$
\begin{aligned}
& \{1,2\} \\
& \{1,4\} \\
& \left(\begin{array}{ccc}
1 & 2 & 4 \\
\left(p_{1}-p_{2}\right)^{T} & \left(p_{2}-p_{1}\right)^{T}\left(\begin{array}{l}
0 \\
1
\end{array}\right) & 0 \\
& 0 & 0
\end{array}\right) \\
& =\left(\begin{array}{ccc}
(a, b-c) & (c-b) & 0 \\
(a, b-d) & 0 & (d-b)
\end{array}\right) .
\end{aligned}
$$

Example 5.3 The orbit rigidity matrix for the framework $\left(K_{2,2}, p\right) \in \mathcal{R}_{\left(K_{2,2}, \mathcal{C}_{s}, \Phi_{b}\right)}$ in Example 4.2 (Fig. 2(b)) is a $3 \times 4$ matrix, since there are three edge orbitsrepresented by the edges $\{1,2\},\{1,4\}$, and $\{2,3\}$, for example-and two vertex orbits-represented by the vertices 1 and 2, for example, and none of the joints of $\left(K_{2,2}, p\right)$ are fixed by the reflection in $\mathcal{C}_{s}$. Note, however, that the end-vertices of the edge $\{1,4\}$ lie in the same vertex orbit for 1 under the action of $\mathcal{C}_{s}$ and that the endvertices of the edge $\{2,3\}$ lie in the same vertex orbit for 2 under the action of $\mathcal{C}_{s}$. Thus, for the orbit rigidity matrix of $\left(K_{2,2}, p\right)$ we write

$$
\begin{aligned}
& 12 \\
& \begin{array}{l}
\{1,2\} \\
\{1,4\} \\
\{2,3\}
\end{array}\left(\begin{array}{cc}
\left(p_{1}-p_{2}\right)^{T} & \left(p_{2}-p_{1}\right)^{T} \\
2\left(p_{1}-s\left(p_{1}\right)\right)^{T} & 00 \\
00 & 2\left(p_{2}-s\left(p_{2}\right)\right)^{T}
\end{array}\right) \\
& 12 \\
& =\left(\begin{array}{cc}
(a-c, b-d) & (c-a, d-b) \\
(4 a, 0) & 00 \\
00 & (4 c, 0)
\end{array}\right) .
\end{aligned}
$$

We now give the general definition of the orbit rigidity matrix of a symmetric framework.

Definition 5.1 Let $G$ be a graph, $S$ be a symmetry group in dimension $d, \Phi: S \rightarrow$ $\operatorname{Aut}(G)$ be a homomorphism, and $(G, p)$ be a framework in $\mathcal{R}_{(G, S, \Phi)}$. Further, let $\mathcal{O}_{V(G)}=\{1, \ldots, k\}$ be a set of representatives for the orbits $S(i)=\{\Phi(x)(i) \mid x \in S\}$ of vertices of $G$. We construct the orbit rigidity matrix (or in short, orbit matrix) $\mathbf{O}(G, p, S)$ of $(G, p)$ so that it has exactly one row for each orbit $S(e)=$ $\{\Phi(x)(e) \mid x \in S\}$ of edges of $G$ and exactly $c_{i}=\operatorname{dim}\left(U\left(p_{i}\right)\right)$ columns for each vertex $i \in \mathcal{O}_{V(G)}$. 
Given an edge orbit $S(e)$ of $G$, there are two possibilities for the corresponding row in $\mathbf{O}(G, p, S)$.

Case 1: The two end-vertices of the edge $e$ lie in distinct vertex orbits. Then there exists an edge in $S(e)$ that is of the form $\{a, x(b)\}$ for some $x \in S$, where $a, b \in$ $\mathcal{O}_{V(G)}$. Let a basis $\mathcal{B}_{a}$ for $U\left(p_{a}\right)$ and a basis $\mathcal{B}_{b}$ for $U\left(p_{b}\right)$ be given and let $\mathbf{M}_{a}$ and $\mathbf{M}_{b}$ be the matrices whose columns are the coordinate vectors of $\mathcal{B}_{a}$ and $\mathcal{B}_{b}$ relative to the canonical basis of $\mathbb{R}^{d}$, respectively. The row we write in $\mathbf{O}(G, p, S)$ is

$$
\left.\begin{array}{ccccc}
a & b \\
0 \ldots 0 & \left(p_{a}-x\left(p_{b}\right)\right)^{T} \mathbf{M}_{a} & 0 \ldots 0 & \left(p_{b}-x^{-1}\left(p_{a}\right)\right)^{T} \mathbf{M}_{b} & 0 \ldots 0
\end{array}\right) .
$$

Case 2: The two end-vertices of the edge $e$ lie in the same vertex orbit. Then there exists an edge in $S(e)$ that is of the form $\{a, x(a)\}$ for some $x \in S$, where $a \in \mathcal{O}_{V(G)}$. Let a basis $\mathscr{B}_{a}$ for $U\left(p_{a}\right)$ be given and let $\mathbf{M}_{a}$ be the matrix whose columns are the coordinate vectors of $\mathcal{B}_{a}$ relative to the canonical basis of $\mathbb{R}^{d}$. The row we write in $\mathbf{O}(G, p, S)$ is:

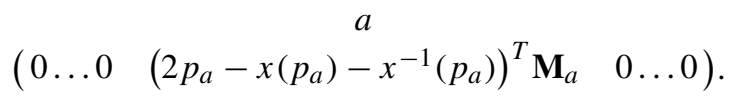

In particular, if $x\left(p_{a}\right)=x^{-1}\left(p_{a}\right)$, this row becomes

$$
\begin{gathered}
a \\
\left(\begin{array}{ll}
0 \ldots 0 & 2\left(p_{a}-x\left(p_{a}\right)\right)^{T} \\
\mathbf{M}_{a} & 0 \ldots 0
\end{array}\right) .
\end{gathered}
$$

Remark 5.1 Note that the rank of the orbit rigidity matrix $\mathbf{O}(G, p, S)$ is clearly independent of the choice of bases for the spaces $U\left(p_{a}\right)$ (and their corresponding matrices $\left.\mathbf{M}_{a}\right), a=1, \ldots, k$.

Remark 5.2 If none of the joints of $(G, p)$ are fixed by any non-trivial symmetry operation in $S$, then the orbit rigidity matrix $\mathbf{O}(G, p, S)$ of $(G, p)$ has $d k=d\left|\mathcal{O}_{V(G)}\right|$ columns, and each of the matrices $\mathbf{M}_{a}$ and $\mathbf{M}_{b}$ may be chosen to be the $d \times d$ identity matrix. In this case, the matrix $\mathbf{O}(G, p, S)$ becomes particularly easy to construct (see Examples 5.1 and 5.3).

\section{The Kernel of $\mathrm{O}(G, p, S)$}

In this section, we show that the kernel of the orbit rigidity matrix $\mathbf{O}(G, p, S)$ of a symmetric framework $(G, p) \in \mathcal{R}_{(G, S, \Phi)}$ is the space of all fully $(S, \Phi)$-symmetric infinitesimal motions of $(G, p)$, restricted to the set $\mathcal{O}_{V(G)}$ of representatives for the vertex orbits $S(i)$ of $G$ (Theorem 6.1). It follows from this result that we can detect whether $(G, p)$ has a fully $(S, \Phi)$-symmetric infinitesimal flex by simply computing the rank of $\mathbf{O}(G, p, S)$. 
Theorem 6.1 Let $G$ be a graph, $S$ be a symmetry group in dimension $d, \Phi: S \rightarrow$ Aut $(G)$ be a homomorphism, $\mathcal{O}_{V(G)}=\{1, \ldots, k\}$ be a set of representatives for the orbits $S(i)=\{\Phi(x)(i) \mid x \in S\}$ of vertices of $G$, and $(G, p) \in \mathcal{R}_{(G, S, \Phi)}$. Further, for each $i=1, \ldots k$, let a basis $\mathcal{B}_{i}$ for $U\left(p_{i}\right)$ be given and let $\mathbf{M}_{i}$ be the $d \times c_{i}$ matrix whose columns are the coordinate vectors of $\mathcal{B}_{i}$ relative to the canonical basis of $\mathbb{R}^{d}$. Then

$$
\tilde{u}=\left(\begin{array}{c}
\tilde{u}_{1} \\
\vdots \\
\tilde{u}_{k}
\end{array}\right) \in \mathbb{R}^{c_{1}} \times \cdots \times \mathbb{R}^{c_{k}}
$$

lies in the kernel of $\mathbf{O}(G, p, S)$ if and only if

$$
\bar{u}=\left(\begin{array}{c}
\mathbf{M}_{1} \tilde{u}_{1} \\
\vdots \\
\mathbf{M}_{k} \tilde{u}_{k}
\end{array}\right) \in \mathbb{R}^{d k}
$$

is the restriction $\left.u\right|_{\mathcal{O}_{V(G)}}$ of a fully $(S, \Phi)$-symmetric infinitesimal motion $u$ of $(G, p)$ to $\mathcal{O}_{V(G)}$.

Proof Suppose there exists an edge $e=\{a, x(b)\}$ in $G$ whose two end-vertices lie in distinct vertex orbits (see Case 1 in the definition of the orbit rigidity matrix). The row equation of the matrix $\mathbf{O}(G, p, S)$ for the edge orbit represented by $e$ is then of the form

$$
\left(p_{a}-\mathbf{X} p_{b}\right)^{T}\left(\mathbf{M}_{a} \tilde{u}_{a}\right)+\left(p_{b}-\mathbf{X}^{-1} p_{a}\right)^{T}\left(\mathbf{M}_{b} \tilde{u}_{b}\right)=0,
$$

where $\mathbf{X}$ is the matrix that represents $x$ with respect to the canonical basis of $\mathbb{R}^{d}$. Since the inner product in the second summand is invariant under the orthogonal transformation $x \in S$, we have

$$
\left(p_{a}-\mathbf{X} p_{b}\right)^{T}\left(\mathbf{M}_{a} \tilde{u}_{a}\right)+\left(\mathbf{X} p_{b}-p_{a}\right)^{T}\left(\mathbf{X M}_{b} \tilde{u}_{b}\right)=0
$$

which is the row equation of the standard rigidity matrix $\mathbf{R}(G, p)$ for $e=\{a, x(b)\}$.

Similarly, for any other edge $y(\{a, x(b)\}), y \in S$, that lies in the edge orbit $S(e)$, we have

$$
\left(\mathbf{Y} p_{a}-\mathbf{Y X} p_{b}\right)^{T}\left(\mathbf{Y} \mathbf{M}_{a} \tilde{u}_{a}\right)+\left(\mathbf{Y X} p_{b}-\mathbf{Y} p_{a}\right)^{T}\left(\mathbf{Y} \mathbf{X} \mathbf{M}_{b} \tilde{u}_{b}\right)=0
$$

where $\mathbf{Y}$ is the matrix that represents $y$ with respect to the canonical basis of $\mathbb{R}^{d}$. This is the standard row equation of $\mathbf{R}(G, p)$ for the edge $y(\{a, x(b)\})$.

Suppose next that there exists a bar $e=\{a, x(a)\}$ in $G$ whose two end-vertices lie in the same vertex orbit (see Case 2 in the definition of the orbit rigidity matrix). The row equation of the matrix $\mathbf{O}(G, p, S)$ for the edge orbit represented by $e$ is then of the form

$$
\left(p_{a}-\mathbf{X} p_{a}\right)^{T}\left(\mathbf{M}_{a} \tilde{u}_{a}\right)+\left(p_{a}-\mathbf{X}^{-1} p_{a}\right)^{T}\left(\mathbf{M}_{a} \tilde{u}_{a}\right)=0 .
$$


Since the inner product in the second summand is invariant under the orthogonal transformation $x \in S$, we have

$$
\left(p_{a}-\mathbf{X} p_{a}\right)^{T}\left(\mathbf{M}_{a} \tilde{u}_{a}\right)+\left(\mathbf{X} p_{a}-p_{a}\right)^{T}\left(\mathbf{X} \mathbf{M}_{a} \tilde{u}_{a}\right)=0,
$$

which is the standard row equation of $\mathbf{R}(G, p)$ for $e=\{a, x(a)\}$.

Similarly, for any other edge $y(\{a, x(a)\}), y \in S$, that lies in the edge orbit $S(e)$, we have

$$
\left(\mathbf{Y} p_{a}-\mathbf{Y X} p_{a}\right)^{T}\left(\mathbf{Y} \mathbf{M}_{a} \tilde{u}_{a}\right)+\left(\mathbf{Y X} p_{a}-\mathbf{Y} p_{a}\right)^{T}\left(\mathbf{Y X} \mathbf{M}_{a} \tilde{u}_{a}\right)=0
$$

which is the standard row equation of $\mathbf{R}(G, p)$ for the edge $y(\{a, x(a)\})$.

It follows that $\tilde{u}$ lies in the kernel of $\mathbf{O}(G, p, S)$ if and only if $\bar{u}$ is the restriction $\left.u\right|_{\mathcal{O}_{V(G)}}$ of a fully $(S, \Phi)$-symmetric infinitesimal motion $u$ of $(G, p)$ to $\mathcal{O}_{V(G)}$.

Example 6.1 Consider the framework $\left(K_{2,2}, p\right) \in \mathcal{R}_{\left(K_{2,2}, \mathcal{C}_{s}, \Phi_{a}\right)}$ from Examples 4.1 and 5.2. The vector $\tilde{u}=\left(-10 \frac{a}{c-b} \frac{a}{d-b}\right)^{T}$ clearly lies in the kernel of $\mathbf{O}\left(K_{2,2}, p, \mathcal{C}_{S}\right)$, and the vector

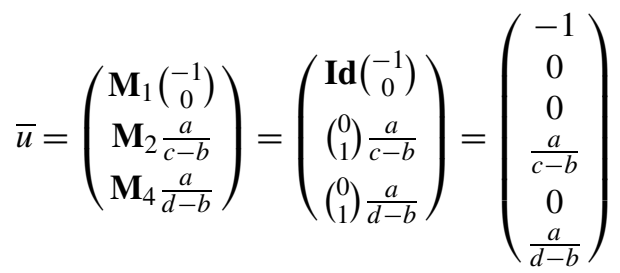

is the restriction of the fully $\left(\mathcal{C}_{s}, \Phi_{a}\right)$-symmetric infinitesimal flex $u=$ $\left(-100 \frac{a}{c-b} 100 \frac{a}{d-b}\right)^{T}$ to the set $\{1,2,4\}$ of representatives for the vertex orbits $\mathcal{C}_{s}(i)=\left\{\Phi_{a}(x)(i) \mid x \in \mathcal{C}_{s}\right\}$.

\section{Symmetry-Preserving Finite Flexes}

The following extension of the theorem of Asimov and Roth (see [2]) to frameworks that possess non-trivial symmetries was derived in [33] (see also [32]):

Theorem 7.1 Let $G$ be a graph, $S$ be a symmetry group in dimension $d, \Phi: S \rightarrow$

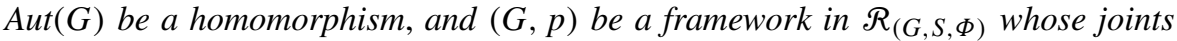
span all of $\mathbb{R}^{d}$. If $(G, p)$ is $(S, \Phi)$-generic and $(G, p)$ has a fully $(S, \Phi)$-symmetric non-trivial infinitesimal motion, then there also exists a mechanism of $(G, p)$ which preserves the symmetry of $(G, p)$ throughout the path.

Remark 7.1 It is also shown in [33] that the condition that $(G, p)$ is $(S, \Phi)$-generic in Theorem 7.1 may be replaced by the weaker condition that the submatrix block $\tilde{\mathbf{R}}_{1}(G, p)$ of the block-diagonalized rigidity matrix $\tilde{\mathbf{R}}(G, p)$ which corresponds to the trivial irreducible representation of $S$ (or, equivalently, the orbit rigidity matrix $\mathbf{O}(G, p, S))$ has maximal rank in some neighborhood of the configuration $p$ within the space of configurations that satisfy the symmetry constraints given by $S$ and $\Phi$. In 
particular, this says that if the rows of the orbit rigidity matrix $\mathbf{O}(G, p, S)$ are linearly independent and $(G, p)$ has a fully $(S, \Phi)$-symmetric infinitesimal flex, then $(G, p)$ also has a symmetry-preserving finite flex.

In combination with Theorem 7.1, Theorem 6.1 gives rise to a simple new method for detecting finite flexes in symmetric frameworks. In the following subsections, we elaborate on this new method and apply it to a number of interesting examples.

\subsection{Detection of Finite Flexes from the Size of the Orbit Rigidity Matrix}

First, we consider situations where knowledge of only the size of the orbit rigidity matrix already allows us to detect finite flexes in symmetric frameworks.

The following result is an immediate consequence of Theorem 6.1:

Theorem 7.2 Let $G$ be a graph, $S$ be a symmetry group in dimension $d, \Phi: S \rightarrow$

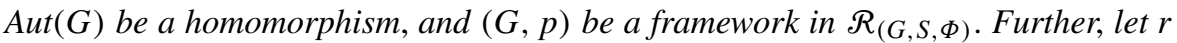
and $c$ denote the number of rows and columns of the orbit rigidity matrix $\mathbf{O}(G, p, S)$, respectively, and let $m$ denote the dimension of the space of fully $(S, \Phi)$-symmetric infinitesimal rigid motions of $(G, p)$. If

$$
r<c-m
$$

then $(G, p)$ has a fully $(S, \Phi)$-symmetric infinitesimal flex.

Recall from Sect. 5 that the number of rows, $r$, and the number of columns, $c$, of the orbit rigidity matrix $\mathbf{O}(G, p, S)$ of $(G, p)$ do not depend on the configuration $p$, but only on the graph $G$ and the prescribed symmetry constraints given by $S$ and $\Phi$. As shown in [32], the dimension $m$ of the space of fully $(S, \Phi)$-symmetric infinitesimal rigid motions of $(G, p)$ is also independent of $p$, provided that the joints of $(G, p)$ span all of $\mathbb{R}^{d}$. So, suppose the set $\mathcal{R}_{(G, S, \Phi)}$ contains a framework whose joints span all of $\mathbb{R}^{d}$. Then, as shown in [35], the joints of all $(S, \Phi)$-generic realizations of $G$ also span all of $\mathbb{R}^{d}$. Thus, if (4) holds, then all $(S, \Phi)$-generic realizations of $G$ have a fully $(S, \Phi)$-symmetric infinitesimal flex, and hence, by Theorem 7.1, also a finite symmetry-preserving flex.

The dimension $m$ of the space of fully $(S, \Phi)$-symmetric infinitesimal rigid motions of $(G, p)$ can easily be computed using the techniques described in [32, 34]. In particular, in dimension 2 and 3, $m$ can be deduced immediately from the character tables given in [14]. Thus, in order to check condition (4) it is only left to determine the size of the orbit rigidity matrix $\mathbf{O}(G, p, S)$ which basically requires only a simple count of the vertex orbits and edge orbits of the graph $G$.

Alternatively, the values of $r$ and $c$ in (4) can also be found by expressing the characters of the 'internal' and 'external' matrix representation for the group $S$ (see [18, $25,34,35]$, for example) as linear combinations of the characters of the irreducible representations of $S$ : the numbers $r$ and $c$ are the respective coefficients corresponding to the trivial irreducible representation in these linear combinations (see [32, 33] for details). However, our new 'orbit approach' is much simpler than this method of computing characters, since it allows us to determine $r$ and $c$ directly without using any techniques from group representation theory. 


\subsubsection{Examples in Dimension 2}

Let us apply our new method to the symmetric quadrilaterals we discussed in Sect. 5.

We first consider the quadrilateral with point group $\mathcal{C}_{2}$ from Example 5.1 (see Fig. 4). There are two vertex orbits, represented by the vertices 1 and 2, for example, and we have $c_{i}=\operatorname{dim}\left(U\left(p_{i}\right)\right)=2$ for $i=1,2$. Further, there are two edge orbits, and we have $m=1$, since the only infinitesimal rigid motions that are fully symmetric with respect to this half-turn symmetry are the ones that correspond to rotations about the origin (see [32] for details). Thus, we have

$$
r=2<3=c-m \text {. }
$$

So, we may conclude that $\left(\mathcal{C}_{2}, \Phi\right)$-generic realizations of $K_{2,2}$ have a symmetrypreserving mechanism (see also Fig. 5(a)).

This result can easily be generalized to predict the flexibility of a whole class of 2-dimensional frameworks with half-turn symmetry.

Recall from Sect. 3 that a joint $p_{i}$ of $(G, p) \in \mathcal{R}_{(G, S, \Phi)}$ is said to be fixed by $x \in S$ if $x\left(p_{i}\right)=p_{i}$. Similarly, we say that a bar $\left\{p_{i}, p_{j}\right\}$ of $(G, p)$ is fixed by $x$ if either $x\left(p_{i}\right)=p_{i}$ and $x\left(p_{j}\right)=p_{j}$ or $x\left(p_{i}\right)=p_{j}$ and $x\left(p_{j}\right)=p_{i}$. The number of joints and bars of $(G, p)$ that are fixed by $x$ are denoted by $j_{x}$ and $b_{x}$, respectively.

Theorem 7.3 Let $G$ be a graph with $|E(G)|=2|V(G)|-4, \mathcal{C}_{2}=\left\{I d, C_{2}\right\}$ be the half-turn symmetry group in dimension 2 , and $\Phi: \mathcal{C}_{2} \rightarrow \operatorname{Aut}(G)$ be a homomorphism. If $j_{C_{2}}=b_{C_{2}}=0$, then $\left(\mathcal{C}_{2}, \Phi\right)$-generic realizations of $G$ have a symmetry-preserving mechanism.

Proof Since $j_{C_{2}}=0$ we have $c=2 \frac{|V(G)|}{2}=|V(G)|$, and since $b_{C_{2}}=0$ we have $r=\frac{|E(G)|}{2}=|V(G)|-2$. As mentioned above, we have $m=1$, so that

$$
r=|V(G)|-2<|V(G)|-1=c-m .
$$

Thus, by Theorems 6.1 and $7.1,\left(\mathcal{C}_{2}, \Phi\right)$-generic realizations of $G$ have a symmetrypreserving mechanism.

Next, we consider the quadrilateral with point group $\mathcal{C}_{s}$ from Example 4.1 (see Fig. 2(a)). There are three vertex orbits, represented by the vertices 1,2 , and 4 , for example, and we have $c_{i}=\operatorname{dim}\left(U\left(p_{i}\right)\right)=1$ for $i=2,4$ and $c_{1}=\operatorname{dim}\left(U\left(p_{1}\right)\right)=2$.

Fig. 5 Symmetry-preserving mechanisms of the quadrilaterals from Examples 5.1 (a) and 4.1 (b)

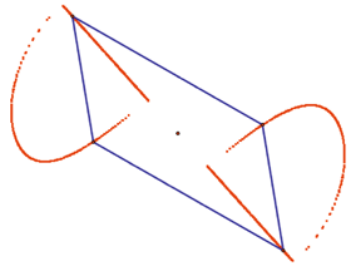

(a)

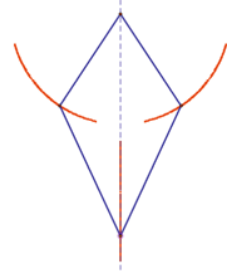

(b) 
Further, there are two edge orbits, and we have $m=1$, since the only infinitesimal rigid motions that are fully symmetric with respect to this mirror symmetry are the ones that correspond to translations along the mirror line [32]. Thus, we have

$$
r=2<3=c-m \text {. }
$$

So, we may conclude that $\left(\mathcal{C}_{s}, \Phi_{a}\right)$-generic realizations of $K_{2,2}$ have a symmetrypreserving mechanism (see also Fig. 5(b)).

The following theorem provides a generalized version of this result.

Theorem 7.4 Let $G$ be a graph with $|E(G)|=2|V(G)|-4, \mathcal{C}_{s}=\{I d$, s $\}$ be a 'reflectional' symmetry group in dimension 2 , and $\Phi: \mathcal{C}_{s} \rightarrow \operatorname{Aut}(G)$ be a homomorphism. If $b_{s}=0$, then $\left(\mathcal{C}_{s}, \Phi\right)$-generic realizations of $G$ have a symmetry-preserving mechanism.

Proof Let $\left|V^{\prime}\right|$ be the number of vertex orbits of size 2 and $\left|E^{\prime}\right|$ be the number of edge orbits of size 2 . Then we have

$$
\begin{aligned}
|E(G)| & =2|V(G)|-4 \\
2\left|E^{\prime}\right|+b_{s} & =2\left(2\left|V^{\prime}\right|+j_{s}\right)-4 \\
\left|E^{\prime}\right|+\frac{1}{2} b_{s} & =2\left|V^{\prime}\right|+j_{s}-2 \\
\left|E^{\prime}\right|+b_{s} & =2\left|V^{\prime}\right|+j_{s}-1+\frac{1}{2} b_{s}-1 \\
r & =c-m+\frac{1}{2} b_{s}-1 .
\end{aligned}
$$

So if $b_{s}<2$ (or, equivalently, $b_{s}=0$, since $b_{s}=1$ contradicts the count $|E(G)|=$ $2|V(G)|-4)$ then $r<c-m$. The result now follows from Theorems 6.1 and 7.1.

Similar results to Theorems 7.3 and 7.4 can of course also be established for other symmetry groups in dimension 2 .

Note that the orbit count for the quadrilateral with mirror symmetry from Example 4.2 (see Fig. 2(b), (c)) is

$$
r=3=c-m,
$$

and by computing the rank of the corresponding orbit rigidity matrix explicitly, it is easy to verify that this quadrilateral does in fact not have any fully $\left(\mathcal{C}_{s}, \Phi_{b}\right)$ symmetric infinitesimal flex, let alone a symmetry-preserving mechanism. However, it does have a mechanism that breaks the mirror symmetry.

\subsubsection{Examples in Dimension 3}

The Bricard octahedra [8] are famous examples of flexible frameworks in 3-space. While it follows from Cauchy's Theorem [9] that convex realizations of the octahedral graph are isostatic, the French engineer R. Bricard found three types of octahedra with self-intersecting faces whose realizations as frameworks are flexible. Two of 
these three types of Bricard octahedra possess non-trivial symmetries: Bricard octahedra of the first type have a half-turn symmetry and Bricard octahedra of the second type have a mirror symmetry. In the following, we use our new 'orbit approach' to not only show that both of these types of frameworks are flexible, but also that they possess a 'symmetry-preserving' finite flex. Various other treatments of the Bricard octahedra can be found in [3, 33, 39], for example. R. Connelly's celebrated counterexample to Euler's rigidity conjecture from 1776 is also based on a flexible Bricard octahedron (of the first type) [10]. However, since the flexible Connelly sphereas well as Steffen's modified Connelly sphere-breaks the half-turn symmetry as it flexes, our methods do not apply to these particular examples.

We let $G$ be the graph of the octahedron, $\mathcal{C}_{2}$ be a 'half-turn' symmetry group in dimension 3, and $\Phi: \mathcal{C}_{2} \rightarrow \operatorname{Aut}(G)$ be the homomorphism defined by

$$
\begin{aligned}
& \Phi(I d)=i d \\
& \Phi\left(C_{2}\right)=(13)(24)(56) .
\end{aligned}
$$

Then there are three vertex orbits-represented by the vertices 1,2 , and 5 , for example (see also Fig. 6(a)). Since none of the joints $p_{1}, p_{2}$, and $p_{5}$ are fixed by the half-turn $C_{2}$, the number of columns of the orbit rigidity matrix $\mathbf{O}\left(G, p, \mathcal{C}_{2}\right)$ is

$$
c=3 \cdot 3=9 \text {. }
$$

Since there are clearly six edge orbits, each of size 2 , we have

$$
r=6 \text {. }
$$

Finally, as shown in [32], we have

$$
m=2 \text {, }
$$

since the fully $\left(\mathcal{C}_{2}, \Phi\right)$-symmetric infinitesimal rigid motions are those that arise from translations along the $C_{2}$-axis and rotations about the $C_{2}$-axis. It follows that

$$
r=6<7=c-m,
$$

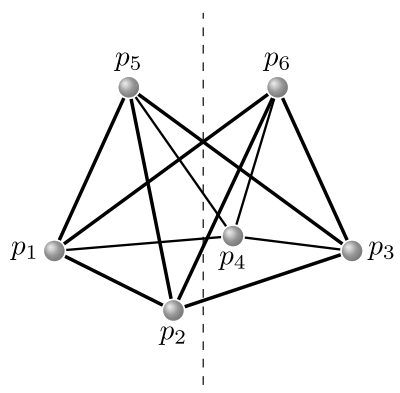

(a)

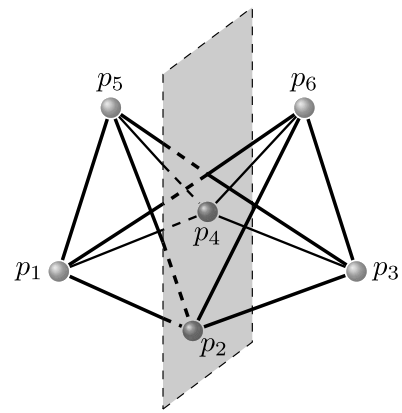

(b)

Fig. 6 Flexible Bricard octahedra with point group $\mathcal{C}_{2}(\mathbf{a})$ and $\mathcal{C}_{S}(\mathbf{b})$ 
so that we may conclude that $\left(\mathcal{C}_{2}, \Phi\right)$-generic realizations of the octahedral graph possess a symmetry-preserving finite flex.

This result can be generalized as follows.

Theorem 7.5 Let $G$ be a graph with $|E(G)|=3|V(G)|-6, \mathcal{C}_{2}=\left\{I d, C_{2}\right\}$ be a halfturn symmetry group in dimension 3 , and $\Phi: \mathcal{C}_{2} \rightarrow A u t(G)$ be a homomorphism. If $j_{C_{2}}=b_{C_{2}}=0$, then $\left(\mathcal{C}_{2}, \Phi\right)$-generic realizations of $G$ have a symmetry-preserving mechanism.

Proof Since $j_{C_{2}}=0$ we have $c=3 \frac{|V(G)|}{2}$, and since $b_{C_{2}}=0$ we have $r=\frac{|E(G)|}{2}$. As mentioned above, we have $m=2$, and hence

$$
r=3 \frac{|V(G)|}{2}-3<3 \frac{|V(G)|}{2}-2=c-m .
$$

So, by Theorems 6.1 and $7.1,\left(\mathcal{C}_{2}, \Phi\right)$-generic realizations of $G$ have a symmetrypreserving mechanism.

The flexibility of the Bricard octahedron with mirror symmetry shown in Fig. 6(b) follows from the following result:

Theorem 7.6 Let $G$ be a graph with $|E(G)|=3|V(G)|-6, \mathcal{C}_{s}=\{I d$, s $\}$ be a reflectional symmetry group in dimension 3 , and $\Phi: \mathcal{C}_{s} \rightarrow$ Aut $(G)$ be a homomorphism. If $j_{s}>b_{s}$, then $\left(\mathcal{C}_{s}, \Phi\right)$-generic realizations of $G$ have a symmetry-preserving mechanism.

Proof Let $\left|V^{\prime}\right|$ be the number of vertex orbits of size 2 and $\left|E^{\prime}\right|$ be the number of edge orbits of size 2 . Then we have

$$
\begin{aligned}
|E(G)| & =3|V(G)|-6 \\
2\left|E^{\prime}\right|+b_{s} & =3\left(2\left|V^{\prime}\right|+j_{s}\right)-6 \\
\left|E^{\prime}\right|+\frac{1}{2} b_{s} & =3\left|V^{\prime}\right|+\frac{3}{2} j_{s}-3 \\
\left|E^{\prime}\right|+b_{s} & =3\left|V^{\prime}\right|+2 j_{s}+\frac{1}{2}\left(b_{s}-j_{s}\right)-3 \\
r & =c+\frac{1}{2}\left(b_{s}-j_{s}\right)-m .
\end{aligned}
$$

So if $j_{s}>b_{s}$, then $r<c-m$. Thus, by Theorems 6.1 and 7.1, $\left(\mathcal{C}_{s}, \Phi\right)$-generic realizations of $G$ have a symmetry-preserving mechanism.

Note that Theorems 7.5 and 7.6 also prove the existence of a symmetry-preserving finite flex in a number of other famous and interesting frameworks in 3-space. For example, Theorem 7.5 applies to symmetric 'double-suspensions' which are frameworks that consist of an arbitrary $2 n$-gon and two 'cone-vertices' that are linked to each of the joints of the $2 n$-gon [12] and to some symmetric ring structures and 
reticulated cylinder structures like the ones analyzed in [20, 42], and [32]. Similarly, Theorem 7.6 applies to the famous 'double-banana' (see [19], for example) with mirror symmetry (with the two connecting vertices of the two 'bananas' lying on the mirror), to various bipartite frameworks (such as 3-dimensional realizations of $K_{4,6}$ with mirror symmetry, where all the joints of either one of the partite sets lie in the mirror [32]), and to some other symmetric ring structures and reticulated cylinder structures.

Similar results to Theorems 7.5 and 7.6 can of course also be established for some other symmetry groups in dimension 3 . In particular, it can be shown that realizations of the octahedral graph which are generic with respect to the dihedral symmetry arising from the half-turn symmetry and the mirror symmetry defined in the examples above also possess a finite flex which preserves the dihedral symmetry throughout the path (see also [32]). Notice that the configurations which are symmetry generic for the dihedral symmetry are not symmetry generic for the half-turn, or the mirror, alone, so this separate analysis is needed.

\subsubsection{Examples in Dimension $d>3$}

Since the orbit counts remain remarkably simple in all dimensions, our new method becomes particularly useful in analyzing symmetric structures in higher dimensional space. We demonstrate this by giving a very simple proof for the flexibility of the 4-dimensional cross-polytope described in [40]. As we will discuss in Sect. 9.3, this example can also immediately be transferred to various other metrics.

For the graph $G$ of the 4-dimensional cross-polytope, we have $|V(G)|=8$ and $|E(G)|=24$, and hence $|E(G)|-(4|V(G)|-10)=24-(4 \cdot 8-10)=2$. Therefore, there will always be at least two linearly independent self-stresses in any 4-dimensional realization of $G$. However, it turns out that certain symmetric 4dimensional cross-polytopes still become flexible: Consider 4-dimensional realizations of $G$ with dihedral symmetry $\mathcal{C}_{2 v}$ which are constructed by placing two joints in each of the two perpendicular mirrors that correspond to the two reflections in $\mathcal{C}_{2 v}$, and adding their mirror reflections, and then connecting each of these eight vertices to all other vertices of $G$ except its own mirror image. This gives rise to four vertex orbits-each of size 2 . Since each mirror is a 3-dimensional hyperplane, we have

$$
c=4 \cdot 3=12 \text {. }
$$

Further, it is easy to check that there are $r=8$ edge orbits ( 4 orbits of size 4 and 4 orbits of size 2) and that $m=3$ (the fully symmetric infinitesimal rigid motions are the ones that arise from translations along the symmetry element (the "plane of rotation') of $C_{2}$ and rotations about the plane perpendicular to the symmetry element of $C_{2}$ ). It follows that

$$
r=8<9=c-m,
$$

which, by Theorems 7.5 and 7.6, implies that 4-dimensional cross-polytopes which are generic with respect to this type of dihedral symmetry possess a symmetrypreserving finite flex. 
Next, we provide some general counting results for frameworks with point groups $\mathcal{C}_{2}$ and $\mathcal{C}_{s}$ in dimension $d>3$ whose underlying graphs satisfy the necessary count $|E(G)|=d|V(G)|-\left(\begin{array}{c}d+1 \\ 2\end{array}\right)$ to be generically isostatic in dimension $d$.

Using the techniques described in [32] it is easy to show that for a $d$-dimensional framework $(G, p)$ with point group $\mathcal{C}_{2}\left(\mathcal{C}_{s}\right)$, the space of fully symmetric infinitesimal translations has dimension $(d-2)(d-1$, respectively) and the space of fully symmetric infinitesimal rotations has dimension $\left(\begin{array}{c}d-2 \\ 2\end{array}\right)+1\left(\left(\begin{array}{c}d-1 \\ 2\end{array}\right)\right.$, respectively), so that the dimension $m$ of fully symmetric infinitesimal rigid motions is equal to $d-2+\left(\begin{array}{c}d-2 \\ 2\end{array}\right)+1=1+\left(\begin{array}{c}d-1 \\ 2\end{array}\right)\left(d-1+\left(\begin{array}{c}d-1 \\ 2\end{array}\right)=\left(\begin{array}{c}d \\ 2\end{array}\right)\right.$, respectively), provided that the joints of $(G, p)$ span all of $\mathbb{R}^{d}$. Alternatively, this can be shown by computing the dimension of the kernel of the corresponding orbit rigidity matrix $\mathbf{O}\left(K_{n}, p, S\right)$, where $K_{n}$ is the complete graph on the vertex set of $G$.

Theorem 7.7 Let $G$ be a graph with $|E(G)|=d|V(G)|-\left(\begin{array}{c}d+1 \\ 2\end{array}\right), \mathcal{C}_{2}=\left\{I d, C_{2}\right\}$ be a half-turn symmetry group in dimension $d$, and $\Phi: \mathcal{C}_{2} \rightarrow \operatorname{Aut}(G)$ be a homomorphism.

(i) If $d=4$ and $b_{C_{2}}=0$, then $\left(\mathcal{C}_{2}, \Phi\right)$-generic realizations of $G$ have a symmetrypreserving mechanism;

(ii) if $d>4$ and $j_{C_{2}}>\frac{b_{C_{2}}}{d-4}+\frac{d(d-7)+8}{2(d-4)}$, then $\left(\mathcal{C}_{2}, \Phi\right)$-generic realizations of $G$ have a symmetry-preserving mechanism.

Proof Let $\left|V^{\prime}\right|$ be the number of vertex orbits of size 2 and $\left|E^{\prime}\right|$ be the number of edge orbits of size 2 .

(i) If $d=4$, then with $b_{C_{2}}=0$ we have

$$
r=2|V(G)|-5<2|V(G)|-4=2\left(2\left|V^{\prime}\right|+j_{s}\right)-4=c-m .
$$

(ii) If $d>4$, then

$$
\begin{aligned}
|E(G)| & =d|V(G)|-\left(\begin{array}{c}
d+1 \\
2
\end{array}\right) \\
2\left|E^{\prime}\right|+b_{C_{2}} & =d\left(2\left|V^{\prime}\right|+j_{C_{2}}\right)-\left(\begin{array}{c}
d+1 \\
2
\end{array}\right) \\
\left|E^{\prime}\right|+b_{C_{2}} & =d\left|V^{\prime}\right|+\frac{d}{2} j_{C_{2}}+\frac{1}{2} b_{C_{2}}-\frac{d(d+1)}{4} \\
r & =\left(d\left|V^{\prime}\right|+(d-2) j_{C_{2}}\right)-\frac{d-4}{2} j_{C_{2}}+\frac{1}{2} b_{C_{2}}-\frac{d(d+1)}{4} \\
r & =c-\frac{d-4}{2} j_{C_{2}}+\frac{1}{2} b_{C_{2}}-\frac{d(d+1)}{4} \\
r & =c+\left(\frac{1}{2} b_{C_{2}}-\frac{d-4}{2} j_{C_{2}}+\frac{d(d-7)}{4}+2\right)-\left(1+\left(\begin{array}{c}
d-1 \\
2
\end{array}\right)\right) \\
r & =c+\left(\frac{1}{2} b_{C_{2}}-\frac{d-4}{2} j_{C_{2}}+\frac{d(d-7)}{4}+2\right)-m .
\end{aligned}
$$


It is now easy to verify that if $j_{C_{2}}>\frac{b_{C_{2}}}{d-4}+\frac{d(d-7)+8}{2(d-4)}$, then $r<c-m$. The result now follows from Theorems 6.1 and 7.1.

In the formula $\left(\frac{1}{2} b_{C_{2}}-\frac{d-4}{2} j_{C_{2}}+\frac{d(d-7)}{4}+2\right)$, the case $d=4, b_{C_{2}}=0$ matches part (i). For $d=5$, the formula becomes $\frac{1}{2}\left(b_{C_{2}}-j_{C_{2}}-1\right)$, so that the count $j_{C_{2}}>b_{C_{2}}$ guarantees the existence of a symmetry-preserving finite flex in a $\left(\mathcal{C}_{2}, \Phi\right)$-generic realization of $G$.

A calculation similar to the one in the proof of Theorem 7.7 provides the following result:

Theorem 7.8 Let $G$ be a graph with $|E(G)|=d|V(G)|-\left(\begin{array}{c}d+1 \\ 2\end{array}\right), \mathcal{C}_{s}=\{I d, s\}$ be a reflectional symmetry group in dimension $d>3$, and $\Phi: \mathcal{C}_{s} \rightarrow$ Aut $(G)$ be a homomorphism. If $j_{s}>\frac{b_{s}}{d-2}+\frac{d(d-3)}{2(d-2)}$, then $\left(\mathcal{C}_{s}, \Phi\right)$-generic realizations of $G$ have a symmetry-preserving mechanism.

\subsubsection{Coning the Counts for $d \geq 3$}

Coning of a framework embeds the framework in one higher dimension, then adds a new vertex attached to all previous vertices. This is a technique that takes the counts for a finite flex in a generic framework in dimension $d$, to the counts for a finite flex of the cone framework in dimension $d+1$ [45]. It is natural to consider how this impacts the counts of the orbit matrix.

First, the symmetry-coning will transfer a point group $S$ in dimension $d$ to a new point group $S^{*}$ in dimension $d+1$ by adding the new vertex on the normal to the origin into the new dimension, extending the axes of any rotations, and the mirrors of any reflections in $S$. With this placement, the cone vertex lies in the symmetry element of each of the symmetry operations in $S^{*}$, and the space of trivial symmetry preserving motions increases by exactly the number of columns added to the orbit matrix by this vertex - call this $m_{0}$. The prior edges and vertices have the same orbits. The new edges from the cone vertex to the prior vertices have orbits corresponding to their end points - that is, one new edge orbit for each of the $k=\left|\mathcal{O}_{V(G)}\right|$ prior vertex orbits. The increase in dimension also adds one column under each vertex orbit.

This process transfers the counts which guaranteed symmetry-preserving finite flexes of the original graph in dimension $d$ to counts which guarantee symmetrypreserving finite flexes of the cone graph in dimension $d+1$. Specifically, if $r<c-m$ then

$$
r^{*}=r+k<c+k+m_{0}-\left(m+m_{0}\right)=c^{*}-m^{*} .
$$

Combined, the orbit matrices and coning provide a powerful tool to construct flexible frameworks in all dimensions.

\subsection{Detection of Finite Flexes from the Rank of the Orbit Rigidity Matrix}

We have seen that the count $r \geq c-m$ is a necessary condition for a symmetric framework $(G, p) \in \mathcal{R}_{(G, S, \Phi)}$ to have no fully $(S, \Phi)$-symmetric infinitesimal flex (Theorem 7.2). However, it is not a sufficient condition, so that if $(G, p)$ satisfies 
the count $r \geq c-m$, we need to compute the actual rank of the orbit rigidity matrix $\mathbf{O}(G, p, S)$ to determine whether $(G, p)$ has a fully $(S, \Phi)$-symmetric infinitesimal flex.

Alternatively, one could use group representation theory to block-diagonalize the rigidity matrix $\mathbf{R}(G, p)$ as described in $[25,34]$, and then compute the rank of the submatrix block which corresponds to the trivial irreducible representation of $S$. This approach, however, requires significantly more work than simply finding the rank of the orbit rigidity matrix.

In the following, we demonstrate the simplicity of our new method for detecting finite flexes via the rank of the orbit rigidity matrix with the help of some examples.

\subsubsection{Examples in Dimension 2}

Consider the complete bipartite graph $K_{4,4}$ with partite sets $\{1,2,3,4\}$ and $\{5,6,7,8\}$. This graph is generically rigid in dimension 2. Moreover, any 2-dimensional realization of $K_{4,4}$ has three linearly independent self-stresses since $\left|E\left(K_{4,4}\right)\right|-$ $\left(2\left|V\left(K_{4,4}\right)\right|-3\right)=16-(2 \cdot 8-3)=3$. However, as we will see, under certain symmetry conditions, 2-dimensional realizations of $K_{4,4}$ become flexible.

Let $\mathcal{C}_{2 v}=\left\{I d, C_{2}, s_{h}, s_{v}\right\}$ be the dihedral symmetry group in dimension 2 generated by the reflections $s_{h}$ and $s_{v}$ whose corresponding mirror lines are the $x$-axis and $y$-axis, respectively, and let $\Phi: \mathcal{C}_{2 v} \rightarrow \operatorname{Aut}\left(K_{4,4}\right)$ be the homomorphism defined by

$$
\begin{aligned}
& \Phi(I d)=i d \\
& \Phi\left(C_{2}\right)=(13)(24)(57)(68) \\
& \Phi\left(s_{h}\right)=(14)(23)(58)(67) \\
& \Phi\left(s_{v}\right)=(12)(34)(56)(78) .
\end{aligned}
$$

A framework $\left(K_{4,4}, p\right)$ in the set $\mathcal{R}_{\left(K_{4,4}, \mathcal{C}_{2 v}, \Phi\right)}$ is depicted in Fig. 7. Let us first compute the size of the orbit rigidity matrix $\mathbf{O}\left(K_{4,4}, p, \mathcal{C}_{2 v}\right)$. There are two vertex orbits-represented by the vertices 1 and 5, for example-and also four edge orbits-represented by the edges $\{1,5\},\{1,6\},\{1,7\}$, and $\{1,8\}$, for example. Since $m$ is clearly equal to zero, and $c_{1}=\operatorname{dim}\left(U\left(p_{1}\right)\right)=2$ and $c_{5}=\operatorname{dim}\left(U\left(p_{5}\right)\right)=2$, we have

$$
r=4=c-m .
$$

Fig. 7 A fully

$\left(\mathcal{C}_{2 v}, \Phi\right)$-symmetric

infinitesimal flex of a framework

in $\mathcal{R}_{\left(K_{4,4}, \mathcal{C}_{2 v}, \Phi\right)}$

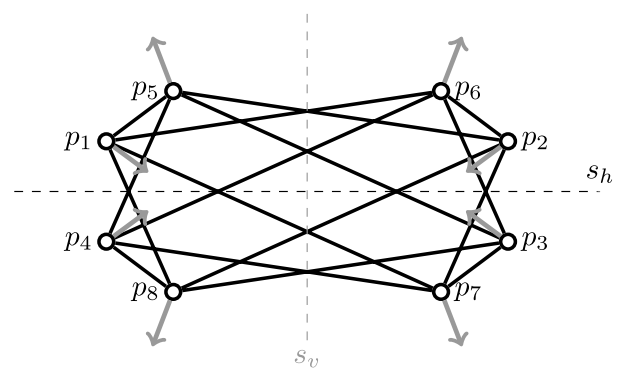


So, to determine whether $\left(K_{4,4}, p\right)$ possesses a fully $\left(\mathcal{C}_{2 v}, \Phi\right)$-symmetric infinitesimal flex, we need to set up the matrix $\mathbf{O}\left(K_{4,4}, p, \mathcal{C}_{2 v}\right)$ explicitly. If we denote $\left(p_{1}\right)^{T}=(a, b)$ and $\left(p_{5}\right)^{T}=(c, d)$, then we have

$$
\begin{aligned}
& \mathbf{O}\left(K_{4,4}, p, \mathcal{C}_{2 v}\right)=\begin{array}{c}
1 \\
\{1,6\} \\
\{1,7\} \\
\{1,8\}
\end{array}\left(\begin{array}{cc}
\left(p_{1}-p_{5}\right)^{T} & \left(p_{5}-p_{1}\right)^{T} \\
\left(p_{1}-s_{v}\left(p_{5}\right)\right)^{T} & \left(p_{5}-s_{v}^{-1}\left(p_{1}\right)\right)^{T} \\
\left(p_{1}-C_{2}\left(p_{5}\right)\right)^{T} & \left(p_{5}-C_{2}^{-1}\left(p_{1}\right)\right)^{T} \\
\left(p_{1}-s_{h}\left(p_{5}\right)\right)^{T} & \left(p_{5}-s_{h}^{-1}\left(p_{1}\right)\right)^{T}
\end{array}\right) \\
& =\left(\begin{array}{ll}
(a-c, b-d) & (c-a, d-b) \\
(a+c, b-d) & (c+a, d-b) \\
(a+c, b+d) & (c+a, d+b) \\
(a-c, b+d) & (c-a, d+b)
\end{array}\right) .
\end{aligned}
$$

It is now easy to see that for any choice of $a, b, c$, and $d$, the rows of $\mathbf{O}\left(K_{4,4}, p, \mathcal{C}_{2 v}\right)$ are linearly dependent (the sum of the first and third row vector minus the sum of the second and fourth row vector is equal to zero). Thus, the kernel of $\mathbf{O}\left(K_{4,4}, p, \mathcal{C}_{2 v}\right)$ is non-trivial, and since $m=0$, it follows that any realization of $K_{4,4}$

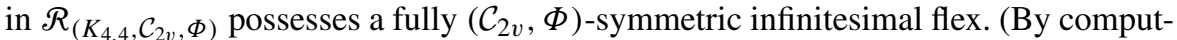
ing an element in the kernel of $\mathbf{O}\left(K_{4,4}, p, \mathcal{C}_{2 v}\right)$ explicitly, it can be seen that all the velocity vectors of this infinitesimal flex are orthogonal to the conic determined by the joints of $\left(K_{4,4}, p\right)$ (see also Fig. 7).) By Theorems 7.5 and 7.6, it follows that $\left(\mathcal{C}_{2 v}, \Phi\right)$-generic realizations of $K_{4,4}$ possess a symmetry-preserving finite flex. This flex is also known as 'Bottema's mechanism [7].

\subsubsection{Examples in Dimension $d>2$}

For any dimension $d>2$, we may construct $d$-dimensional realizations of the complete bipartite graph $K_{2 d, 2 d}$ with point group $\mathcal{C}_{d h}$ by choosing one vertex from each of the two partite sets of $K_{2 d, 2 d}$ as representatives for the vertex orbits and placing them 'generically' off the mirror plane corresponding to the reflection in $\mathcal{C}_{d h}$ and also off the rotational axis corresponding to the $d$-fold rotation in $\mathcal{C}_{d h}$. This gives rise to two vertex orbits (each of size $2 d$ ) and $2 d$ edge orbits (see also Fig. 8). Since the infinitesimal rigid motions corresponding to rotations about the $d$-fold axis will always be fully-symmetric with respect to this $\mathcal{C}_{d h}$ symmetry, the orbit counts will always detect a fully-symmetric self-stress, but no fully-symmetric infinitesimal flex for these frameworks. However, these frameworks can be shown to possess a symmetry-preserving finite flex by computing the actual ranks of the corresponding orbit matrices.

Note that for these types of realizations of $K_{2 d, 2 d}$ with $\mathcal{C}_{d h}$ symmetry, the geometry of quadric surfaces (see $[5,47]$, for example) can be used to predict the existence of a fully-symmetric infinitesimal flex and therefore also the rank properties of the corresponding orbit matrices. 


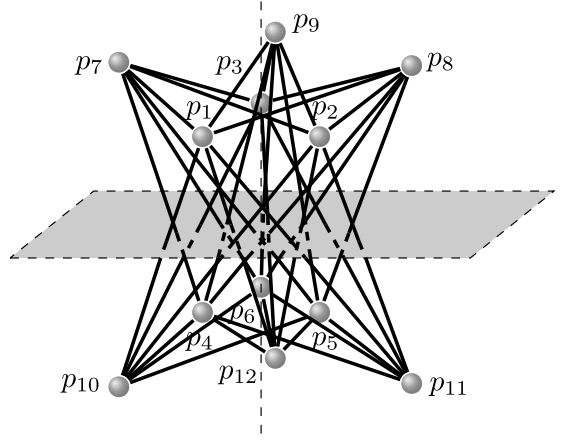

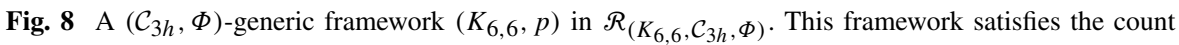
$r=6>5=6-1=c-m$. Since the orbit matrix of $\left(K_{6,6}, p\right)$ is easily seen to have two independent row dependencies, it follows that $\left(K_{6,6}, p\right)$ has a fully $\left(\mathcal{C}_{3 h}, \Phi\right)$-symmetric infinitesimal flex, and hence also a symmetry-preserving finite flex. This example can be thought of as the 3-dimensional analog of Bottema's mechanism

\section{Fully Symmetric Self-Stresses}

\subsection{The Kernel of $\mathbf{O}(G, p, S)^{T}$}

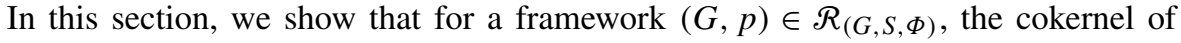
the orbit rigidity matrix $\mathbf{O}(G, p, S)$ is the space of all fully $(S, \Phi)$-symmetric selfstresses of $(G, p)$, restricted to the corresponding set $\mathcal{O}_{E(G)}$ of representatives for the edge orbits $S(e)=\{\Phi(x)(e) \mid x \in S\}$ of $G$ (Theorem 8.3). To prove this result, we need the following two lemmas:

Lemma 8.1 Let $G$ be a graph, $S$ be a symmetry group in dimension $d, \Phi$ : $S \rightarrow \operatorname{Aut}(G)$ be a homomorphism, and $(G, p) \in \mathcal{R}_{(G, S, \Phi)}$. Further, let $S(e)=$ $\{\Phi(x)(e) \mid x \in S\}$ be an edge orbit of $G$ whose representative $e=\{a, x(b)\}$ is an edge whose end-vertices lie in distinct vertex orbits of $G$. Then there exist respective bases $\mathscr{B}_{a}$ and $\mathscr{B}_{b}$ for $U\left(p_{a}\right)$ and $U\left(p_{b}\right)$ (whose coordinate column vectors relative to the canonical basis form the $d \times c_{a}$ matrix $\mathbf{M}_{a}$ and the $d \times c_{b}$ matrix $\mathbf{M}_{b}$, respectively), a scalar $\alpha_{e} \in \mathbb{R}$, and two invertible $d \times d$ matrices $\mathbf{A}$ and $\mathbf{B}$ such that

$$
\sum_{j:\{a, j\} \in S(e)}\left(p_{a}-p_{j}\right)^{T}=\frac{1}{\alpha_{e}}\left(\left(p_{a}-x\left(p_{b}\right)\right)^{T} \mathbf{M}_{a}, 0, \ldots, 0\right) \mathbf{A}
$$

and

$$
\sum_{j:\{b, j\} \in S(e)}\left(p_{b}-p_{j}\right)^{T}=\frac{1}{\alpha_{e}}\left(\left(p_{b}-x^{-1}\left(p_{a}\right)\right)^{T} \mathbf{M}_{b}, 0, \ldots, 0\right) \mathbf{B} .
$$

Proof Let $\left\{I d=y_{0}, y_{1}, \ldots, y_{t}\right\}$ be the stabilizer $S_{p_{a}}=\left\{x \in S \mid x\left(p_{a}\right)=p_{a}\right\}$ of $p_{a}, \mathbf{Y}_{l}$ be the matrix that represents $y_{l}$ with respect to the canonical basis of $\mathbb{R}^{d}$ for each $l$, 
and $\alpha_{e}=\left|S_{p_{a}} \cap S_{x\left(p_{b}\right)}\right|$. Then we have

$$
\sum_{j:\{a, j\} \in S(e)}\left(p_{a}-p_{j}\right)^{T}=\frac{1}{\alpha_{e}} \sum_{l=0}^{t}\left(\mathbf{Y}_{l}\left(p_{a}-x\left(p_{b}\right)\right)\right)^{T},
$$

because

$$
\left(y_{l}\left(\left\{p_{a}, x\left(p_{b}\right)\right\}\right)\right)_{l=0, \ldots, t}
$$

is the family of those bars of $(G, p)$ whose corresponding edges lie in $S(e)$ and are incident with $a$, and because $p_{a}-x\left(p_{b}\right)=y_{l}\left(p_{a}-x\left(p_{b}\right)\right)$ if and only if $y_{l}$ is an element of the coset $S_{p_{a}} \cap S_{x\left(p_{b}\right)}$ of $S_{p_{a}}$.

Consider the matrix representation $H: S_{p_{a}} \rightarrow G L\left(\mathbb{R}^{d}\right)$ that assigns to each $y_{l} \in$ $S_{p_{a}}$ the $d \times d$ matrix $\mathbf{Y}_{l}$ which represents $y_{l}$ with respect to the canonical basis of $\mathbb{R}^{d}$. By definition, the $H$-invariant subspace $\mathbb{V}$ of $\mathbb{R}^{d}$ corresponding to the trivial irreducible representation of $H$ is the space $U\left(p_{a}\right)$. Thus, by the Great Orthogonality Theorem, there exists an orthogonal $d \times d$ matrix of basis transformation $\mathbf{M}$ (i.e., $\mathbf{M}^{-1}=\mathbf{M}^{T}$ ) such that

$$
\sum_{l=0}^{t} \mathbf{M}^{-1} \mathbf{Y}_{l} \mathbf{M}=\mathbf{M}^{-1}\left(\sum_{l=0}^{t} \mathbf{Y}_{l}\right) \mathbf{M}=\left(\begin{array}{cc|c}
t & & \\
& \ddots & \mathbf{0} \\
& t & \\
\hline & \mathbf{0} & \mathbf{0}
\end{array}\right)
$$

where the first $c_{a}$ column vectors of $\mathbf{M}$ are the coordinate vectors of a basis for $\mathbb{V}=U\left(p_{a}\right)$ relative to the canonical basis of $\mathbb{R}^{d}$. We let $\mathbf{M}_{a}$ be such that

$$
\mathbf{M}=\frac{1}{t}\left(\mathbf{M}_{a} \mid \begin{array}{ccc}
\vdots & & \vdots \\
& \ldots & * \\
\vdots & & \vdots
\end{array}\right)
$$

Then, for $\mathbf{A}=\mathbf{M}^{T}$, we have

$$
\left.\begin{array}{rl}
\alpha_{e} \sum_{j:\{a, j\} \in S(e)}\left(p_{a}-p_{j}\right)^{T} & =\left(\left(\sum_{l=0}^{t} \mathbf{Y}_{l}\right)\left(p_{a}-x\left(p_{b}\right)\right)\right)^{T} \\
& =\left(\begin{array}{ll|l}
t & & \\
& \ddots & \mathbf{0} \\
& & \\
& &
\end{array}\right) \mathbf{M}^{T}\left(p_{a}-x\left(p_{b}\right)\right)
\end{array}\right)
$$




$$
\begin{aligned}
& =\left(\mathbf{M}\left(\begin{array}{ccc} 
& \mathbf{M}_{a}^{T} & \\
\cdots & 0 & \ldots \\
& \vdots & \\
\ldots & 0 & \ldots
\end{array}\right)\left(p_{a}-x\left(p_{b}\right)\right)\right)^{T} \\
& =\left(\left(p_{a}-x\left(p_{b}\right)\right)^{T} \mathbf{M}_{a}, 0, \ldots, 0\right) \mathbf{A} \text {. }
\end{aligned}
$$

This proves (5).

Note that if we can show that $\left|S_{p_{a}} \cap S_{x\left(p_{b}\right)}\right|=\left|S_{p_{b}} \cap S_{x^{-1}\left(p_{a}\right)}\right|$, then the proof of (6) proceeds completely analogously to the one of (5).

Consider the map

$$
\psi:\left\{\begin{aligned}
S_{p_{a}} \cap S_{x\left(p_{b}\right)} & \rightarrow S_{p_{b}} \cap S_{x^{-1}\left(p_{a}\right)}, \\
y & \mapsto x^{-1} y x
\end{aligned}\right.
$$

We show that $\psi$ is well-defined. Note that $S_{x\left(p_{b}\right)}=x S_{p_{b}} x^{-1}$ and $S_{x^{-1}\left(p_{a}\right)}=$ $x^{-1} S_{p_{a}} x$. Thus, $y \in S_{p_{a}} \cap S_{x\left(p_{b}\right)}$ if and only if $y \in S_{p_{a}}$ and $y=x \hat{y} x^{-1}$ with $\hat{y} \in$ $S_{p_{b}}$. We have $\psi(y)=x^{-1} y x=x^{-1}\left(x \hat{y} x^{-1}\right) x=\hat{y} \in S_{p_{b}}$, and hence $\psi(y) \in S_{p_{b}} \cap$ $S_{x^{-1}\left(p_{a}\right)}$. Since $\psi$ is clearly bijective, we indeed have $\left|S_{p_{a}} \cap S_{x\left(p_{b}\right)}\right|=\left|S_{p_{b}} \cap S_{x^{-1}\left(p_{a}\right)}\right|$. This gives the result.

Lemma 8.2 Let $G$ be a graph, $S$ be a symmetry group in dimension $d, \Phi: S \rightarrow$

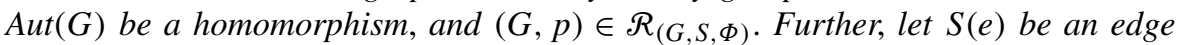
orbit of $G$ whose representative $e=\{a, x(a)\}$ is an edge whose end-vertices lie in the same vertex orbit of $G$. Then there exists a basis $\mathcal{B}_{a}$ for $U\left(p_{a}\right)$ (whose coordinate column vectors relative to the canonical basis form the $d \times c_{a}$ matrix $\mathbf{M}_{a}$ ), a scalar $\alpha_{e} \in \mathbb{R}$, and an invertible $d \times d$ matrix $\mathbf{A}$ such that

$$
\sum_{j:\{a, j\} \in S(e)}\left(p_{a}-p_{j}\right)^{T}=\frac{1}{\alpha_{e}}\left(\left(2 p_{a}-x\left(p_{a}\right)-x^{-1}\left(p_{a}\right)\right)^{T} \mathbf{M}_{a}, 0, \ldots, 0\right) \mathbf{A} .
$$

Proof Let $\left\{I d=y_{0}, y_{1}, \ldots, y_{t}\right\}$ be the stabilizer $S_{p_{a}}=\left\{x \in S \mid x\left(p_{a}\right)=p_{a}\right\}$ of $p_{a}$, and let $\mathcal{F}_{1}$ and $\mathcal{F}_{2}$ be the families of bars of $(G, p)$ defined by

$$
\begin{aligned}
& \mathcal{F}_{1}=\left(y_{l}\left(\left\{p_{a}, x\left(p_{a}\right)\right\}\right)\right)_{l=0, \ldots, t}, \\
& \mathcal{F}_{2}=\left(y_{l}\left(\left\{p_{a}, x^{-1}\left(p_{a}\right)\right\}\right)\right)_{l=0, \ldots, t} .
\end{aligned}
$$

The range of the families $\mathcal{F}_{1}$ and $\mathcal{F}_{2}$ are the bars that correspond to the summands in the left hand side of (7). Note that we either have $\mathcal{F}_{1}=\mathcal{F}_{2}$ or $\mathcal{F}_{1} \cap \mathcal{F}_{2}=\emptyset\left(\mathcal{F}_{1}=\mathcal{F}_{2}\right.$ if and only if there exists $y_{l} \in S_{p_{a}}$ such that $\left.y_{l}\left(x\left(p_{a}\right)\right)=x^{-1}\left(p_{a}\right)\right)$. Moreover, we have $\left|S_{p_{a}} \cap S_{x\left(p_{a}\right)}\right|=\left|S_{p_{a}} \cap S_{x^{-1}\left(p_{a}\right)}\right|$ since, by a similar argument as in the proof of Lemma 8.1, the map

$$
\psi:\left\{\begin{aligned}
S_{p_{a}} \cap S_{x\left(p_{a}\right)} & \rightarrow S_{p_{a}} \cap S_{x^{-1}\left(p_{a}\right)}, \\
y & \mapsto x^{-1} y x
\end{aligned}\right.
$$

is well-defined and bijective. 
Suppose first that $\mathcal{F}_{1} \cap \mathcal{F}_{2}=\emptyset$. Then we have

$$
\begin{aligned}
& \sum_{j:\{a, j\} \in S(e)}\left(p_{a}-p_{j}\right)^{T} \\
& =\frac{1}{\alpha_{e}}\left(\sum_{l=0}^{t}\left(\mathbf{Y}_{l}\left(p_{a}-x\left(p_{a}\right)\right)\right)^{T}+\sum_{l=0}^{t}\left(\mathbf{Y}_{l}\left(p_{a}-x^{-1}\left(p_{a}\right)\right)\right)^{T}\right) \\
& =\frac{1}{\alpha_{e}}\left(\left(\sum_{l=0}^{t} \mathbf{Y}_{l}\right)\left(2 p_{a}-x\left(p_{a}\right)-x^{-1}\left(p_{a}\right)\right)\right)^{T},
\end{aligned}
$$

where $\mathbf{Y}_{l}$ is the matrix that represents $y_{l}$ with respect to the canonical basis of $\mathbb{R}^{d}$ for each $l$, and $\alpha_{e}=\left|S_{p_{a}} \cap S_{x\left(p_{a}\right)}\right|=\left|S_{p_{a}} \cap S_{x^{-1}\left(p_{a}\right)}\right|$.

Suppose next that $\mathcal{F}_{1}=\mathscr{F}_{2}$. Then

$$
\sum_{l=0}^{t}\left(\mathbf{Y}_{l}\left(p_{a}-x\left(p_{a}\right)\right)\right)^{T}=\sum_{l=0}^{t}\left(\mathbf{Y}_{l}\left(p_{a}-x^{-1}\left(p_{a}\right)\right)\right)^{T}
$$

and hence

$$
\sum_{j:\{a, j\} \in S(e)}\left(p_{a}-p_{j}\right)^{T}=\frac{1}{\alpha_{e}}\left(\left(\sum_{l=0}^{t} \mathbf{Y}_{l}\right)\left(2 p_{a}-x\left(p_{a}\right)-x^{-1}\left(p_{a}\right)\right)\right)^{T},
$$

where $\alpha_{e}=2\left|S_{p_{a}} \cap S_{x\left(p_{a}\right)}\right|$.

Now, by the same argument as in the proof of Lemma 8.1, it follows from (8) and (9) that for the scalars $\alpha_{e}$ defined above and the matrices $\mathbf{M}_{a}$ and $\mathbf{A}$ defined in Lemma 8.1, (7) holds.

Theorem 8.3 Let $G$ be a graph with $V(G)=\{1, \ldots, n\}, S$ be a symmetry group in dimension $d, \Phi: S \rightarrow \operatorname{Aut}(G)$ be a homomorphism, $\mathcal{O}_{V(G)}=\{1, \ldots, k\}$ and $\mathcal{O}_{E(G)}=$ $\left\{e_{1}, \ldots, e_{r}\right\}$ be sets of representatives for the vertex orbits $S(i)=\{\Phi(x)(i) \mid x \in S\}$ and edge orbits $S(e)=\{\Phi(x)(e) \mid x \in S\}$ of $G$, respectively, and $(G, p) \in \mathcal{R}_{(G, S, \Phi)}$. If the scalars $\alpha_{e_{i}}, i=1, \ldots, r$, and the bases for the spaces $U\left(p_{i}\right), i=1, \ldots, k$, are defined as in Lemmas 8.1 and 8.2, then $\tilde{\omega} \in \mathbb{R}^{r}$ is an element of the kernel of $\mathbf{O}(G, p, S)^{T}$ if and only if

$$
\bar{\omega}=\left(\begin{array}{c}
\alpha_{e_{1}}(\tilde{\omega})_{1} \\
\vdots \\
\alpha_{e_{r}}(\tilde{\omega})_{r}
\end{array}\right)
$$

is the restriction $\left.\omega\right|_{\mathcal{O}_{E(G)}}$ of a fully $(S, \Phi)$-symmetric self-stress $\omega$ of $(G, p)$ to $\mathcal{O}_{E(G)}$.

Proof We let $\mathbf{O}_{i, a}$ be the $c_{a}$-dimensional row vector which consists of those components of the $i$ th row of $\mathbf{O}(G, p, S)$ that correspond to the vertex $a \in V(G)$. We further let $\overline{\mathbf{O}}_{i, a}$ be the $d$-dimensional row vector $\left(\mathbf{O}_{i, a}, 0, \ldots, 0\right)$. 
Suppose first that $\bar{\omega}$ is the restriction $\left.\omega\right|_{\mathcal{O}_{E(G)}}$ of a fully $(S, \Phi)$-symmetric selfstress $\omega$ of $(G, p)$. Then for every vertex $a=1, \ldots, k$, we have

$$
\sum_{i=1}^{r} \sum_{j:\{a, j\} \in S\left(e_{i}\right)}(\bar{\omega})_{i}\left(p_{a}-p_{j}\right)^{T}=0^{T} .
$$

By (5), (6), and (7), for every vertex $a=1, \ldots, k$, we have

$$
\begin{aligned}
\sum_{i=1}^{r} \sum_{j:\{a, j\} \in S\left(e_{i}\right)}(\bar{\omega})_{i}\left(p_{a}-p_{j}\right)^{T} & =\sum_{i=1}^{r}(\bar{\omega})_{i} \sum_{j:\{a, j\} \in S\left(e_{i}\right)}\left(p_{a}-p_{j}\right)^{T} \\
& =\sum_{i=1}^{r}(\tilde{\omega})_{i}\left(\overline{\mathbf{O}}_{i, a} \mathbf{A}\right) \\
& =\left(\sum_{i=1}^{r}(\tilde{\omega})_{i} \overline{\mathbf{O}}_{i, a}\right) \mathbf{A},
\end{aligned}
$$

where $\mathbf{A}$ is defined as in Lemmas 8.1 and 8.2. (In particular, if $p_{a}$ is not fixed by any non-trivial symmetry operation in $S$, then $\mathbf{A}$ is the $d \times d$ identity matrix and $\overline{\mathbf{O}}_{i, a}=\mathbf{O}_{i, a}$.) Since $\mathbf{A}$ is invertible, it follows that

$$
\sum_{i=1}^{r}(\tilde{\omega})_{i} \overline{\mathbf{O}}_{i, a}=0^{T},
$$

and hence

$$
\sum_{i=1}^{r}(\tilde{\omega})_{i} \mathbf{O}_{i, a}=0^{T}
$$

Conversely, if $\tilde{\omega}$ is an element of the kernel of $\mathbf{O}(G, p, S)^{T}$, then for every vertex $a=1, \ldots, k$, we have

$$
\sum_{i=1}^{r}(\tilde{\omega})_{i} \mathbf{O}_{i, a}=0^{T}
$$

and hence, by the same argument as above,

$$
\sum_{i=1}^{r} \sum_{j:\{a, j\} \in S\left(e_{i}\right)}(\bar{\omega})_{i}\left(p_{a}-p_{j}\right)^{T}=0^{T} .
$$

Moreover, for every $x \in S$, we have

$$
\begin{aligned}
\sum_{i=1}^{r} \sum_{j:\{a, j\} \in S\left(e_{i}\right)}(\bar{\omega})_{i}\left(\mathbf{X}\left(p_{a}-p_{j}\right)\right)^{T} & =\left(\sum_{i=1}^{r} \sum_{j:\{a, j\} \in S\left(e_{i}\right)}(\bar{\omega})_{i}\left(p_{a}-p_{j}\right)^{T}\right) \mathbf{X}^{T} \\
& =0^{T} \mathbf{X}^{T} \\
& =0^{T},
\end{aligned}
$$


where $\mathbf{X}$ is the matrix that represents $x$ with respect to the canonical basis of $\mathbb{R}^{d}$. This completes the proof.

\subsection{Fully Symmetric Tensegrities}

It is natural to investigate how stressed symmetric frameworks can convert to tensegrity frameworks, with cables (members that can get shorter but not longer), struts (members that can get longer but not shorter) as well as bars (whose length is fixed) [30]. A number of the classical tensegrity frameworks are based on symmetric frameworks, and the Robert Connelly's web site [11] permits an interactive exploration of a range of examples of symmetric tensegrity frameworks.

We give a few basic definitions and translate some standard results to the symmetric setting.

A tensegrity graph $\hat{G}$ has a partition of the edges of $G$ into three disjoint parts $E(G)=E_{+}(G) \cup E_{-}(G) \cup E_{0}(G) . E_{+}(G)$ are the edges that are cables, $E_{-}(G)$ are the struts and $E_{0}(G)$ are the bars. For a tensegrity framework $(\hat{G}, p)$, a proper selfstress is a self-stress on the underlying framework $(G, p)$ with the added condition that $\omega_{i j} \geq 0,\{i, j\} \in E_{+}, \omega_{i j} \leq 0,\{i, j\} \in E_{-}[30]$.

Given a symmetric framework $(G, p) \in \mathcal{R}_{(G, S, \Phi)}$, it is possible to use a fully $(S, \Phi)$-symmetric self-stress on the bar and joint framework $(G, p)$ to investigate both the infinitesimal rigidity of $(G, p)$, and the infinitesimal rigidity of an associated fully symmetric tensegrity framework $(\hat{G}, p)$ (i.e., the edges of an edge orbit are either all cables, or all struts, or all bars), with all members with $\omega_{i j}>0$ as cables and all members with $\omega_{i j}<0$ as struts.

The standard result for the infinitesimal rigidity of such frameworks is

Theorem 8.4 (Roth, Whiteley [30]) A tensegrity framework $(\hat{G}, p)$ is infinitesimally rigid if and only if the underlying bar framework $(G, p)$ is infinitesimally rigid as a bar and joint framework and $(G, p)$ has a self-stress which has $\omega_{i j}>0$ on cables and $\omega_{i j}<0$ on struts.

Translated in terms of the orbit matrix for a symmetric framework, this says:

Corollary 8.5 A fully symmetric tensegrity framework $(\hat{G}, p)$ is infinitesimally rigid if and only if the underlying bar framework $(G, p) \in \mathcal{R}_{(G, S, \Phi)}$ is infinitesimally rigid as a bar and joint framework and the orbit matrix $\mathbf{O}(G, p, S)$ has a self-stress which has $\omega_{i j}>0$ on cables and $\omega_{i j}<0$ on struts.

Often, tensegrity frameworks are built which are rigid, but not infinitesimally rigid $[11,13]$. Clearly, the underlying framework $(G, p)$ is not generic (where rigidity is equivalent to infinitesimal rigidity), so $(G, p)$ has some self-stress. The results of Connelly [13] tell us that $(\hat{G}, p)$ has a non-zero proper self-stress.

Theorem 8.6 (Connelly [13], Theorem 3$)$ Let $(\hat{G}, p)$ be a rigid tensegrity framework with a cable or strut. Then there is a proper self-stress in the tensegrity framework (with $\omega_{i j}>0$ on cables and $\omega_{i j}<0$ on struts). 
Given a fully symmetric rigid tensegrity framework $(\hat{G}, p)$, we can show that the guaranteed self-stress can be chosen to be fully symmetric.

Corollary 8.7 Let $(\hat{G}, p)$ be a fully symmetric rigid tensegrity framework with a

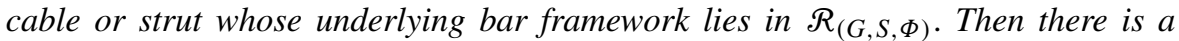
fully $(S, \Phi)$-symmetric non-zero proper self-stress in the tensegrity framework (with $\omega_{i j}>0$ on cables and $\omega_{i j}<0$ on struts $)$.

Proof By Theorem 8.6, there is a non-zero proper self-stress in $(\hat{G}, p)$. We want to symmetrize this self-stress. For each element of the group $x \in S$, and each edge $\{i, j\}$, we have the coefficient $\omega_{x(i, j)}$ of the corresponding element of the orbit. If we add over all elements of the group, this is a finite sum, and we have a combined coefficient $\omega_{S(i, j)}$. It is a direct computation to confirm that these coefficients are a self-stress (the sum of self-stresses is a self-stress) and that they form a fully symmetric self-stress. Since the original stress was proper on a fully symmetric tensegrity framework, all the $\omega_{x(i, j)}$ for a given edge have the same sign, so there is no cancelation. We conclude that this is the required non-zero proper fully symmetric self-stress.

The following example illustrates how these pieces fit together in the layers of symmetry-preserving finite flexes in symmetry generic configurations, nonsymmetric finite flexes for symmetry generic configurations for a larger group, and fully symmetric stresses giving rigidity for an even larger group.

Example 8.1 Consider the graph and frameworks illustrated in Fig. 9.

(a) Figure 9(a) shows the graph $G$ realized at a generic configuration with $\mathcal{C}_{2}$ symmetry. The counts for the rank of the orbit matrix are: $r=6, c=8$ and $m=1$. This guarantees a symmetry-preserving finite flex. While it is not immediate, the standard result for such planar graphs [17] shows that this framework only has a self-stress if it is the projection of a plane faced polyhedron-which this is not (there is no consistent line of intersection of the outside quadrilateral and the inside face). The symmetry-preserving finite flex is also the flex guaranteed by the

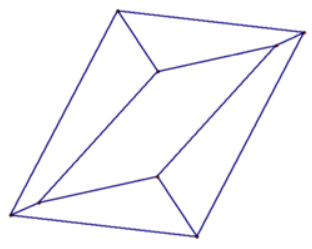

(a)

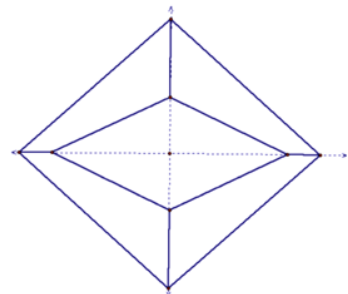

(b)

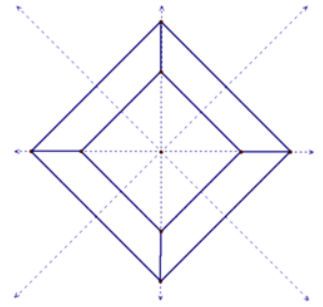

(c)

Fig. 9 We give three plane configurations for the edge graph of a cube. In (a) there is $\mathcal{C}_{2}$ symmetry, in (b) there is $\mathcal{C}_{2 v}$ symmetry, and in (c) there is $\mathcal{C}_{4 v}$ symmetry. (a) has a symmetry-preserving finite flex, (b) has a finite flex which breaks the mirror symmetry, and (c) has a fully symmetric self-stress which makes it rigid 
basic generic counts: $|E(G)|=12,2|V(G)|=16$ and $|E(G)|=12<16-3=$ $2|V(G)|-3$.

(b) Figure 9(b) shows a symmetry generic configuration for $\mathcal{C}_{2 v}$. The new counts are $r=4, c=4$ and $m=0$. The corresponding orbit matrix counts to be independent - and in fact the framework still has no self-stress. The framework is still not the projection of a plane faced polyhedron. There is a finite flex, but it is not symmetry-preserving for this $\mathcal{C}_{2 v}$ symmetry (only for $\mathcal{C}_{2}$ ).

(c) Figure 9(c) shows a symmetry generic configuration for $\mathcal{C}_{4 v}$. The revised counts are: $r=3, c=2$ and $m=0$. We are guaranteed a fully symmetric self-stress. (One can also see this as the projection of a plane faced cube-line polyhedron.) We know that this is rigid (and remains rigid with cables and struts following the signs of the self-stress). With cables on the interior of the framework, this is a spider web, and the approach of [13] just works to confirm that these are rigid (though not infinitesimally rigid).

As the example illustrates, and the many structures on [11] confirm, a fully symmetric self-stress can be the way of forming a rigid tensegrity framework which is too undercounted to be infinitesimally rigid.

We conjecture that a further analog of Connelly's Theorem also holds, and that the basic proof can be symmetry adapted:

Conjecture 8.8 Let $(\hat{G}, p)$ be a fully symmetric tensegrity framework with a cable or strut which has no symmetry-preserving finite flex. Then there is a fully symmetric non-zero proper self-stress in the tensegrity framework (with $\omega_{i j}>0$ on cables and $\omega_{i j}<0$ on struts $)$.

\section{Further Work}

As mentioned in the introduction, the analysis of the orbit matrix opens up a number of questions which are analogs of the previous work for the standard rigidity matrix. The following samples are not exhaustive, and we find new possibilities keep opening up for us as we continue to work with the tools and reflect on the possibilities.

\subsection{Necessary and Sufficient Conditions for a Full Rank Orbit Matrix}

An important question for the standard rigidity matrix has been deriving necessary and sufficient conditions on the graph for the rigidity matrix to be of full rank (generic rigidity), or independent, or to have a self-stress. The most famous example is Laman's Theorem characterizing generic rigidity in the plane [23]. Within the context of symmetric frameworks, there are generalizations for key plane groups $\left(\mathcal{C}_{3}\right.$, $\mathcal{C}_{s}$, and $\mathcal{C}_{2}$ ) presented in $[32,36]$. With these combinatorial calculations come fast algorithms for verifying the rigidity in symmetry-generic configurations.

It is natural to seek necessary and sufficient conditions for the orbit matrix of $(G, p) \in \mathcal{R}_{(G, S, \Phi)}$ to be of full rank (i.e., for $(G, p)$ to have only trivial fully $(S, \Phi)$ symmetric infinitesimal motions) for a symmetry generic $p$, or to be independent 
(i.e., for $(G, p)$ to have no fully $(S, \Phi)$-symmetric self-stresses). Of course, given a symmetric framework $(G, p) \in \mathcal{R}_{(G, S, \Phi)}$ which is independent and infinitesimally rigid with the usual rigidity matrix, its orbit matrix will also be independent and of maximal rank. However, we have seen that there are frameworks which are dependent but the lack of a fully $(S, \Phi)$-symmetric self stress means that the orbit matrix is independent, as well as frameworks which have infinitesimal flexes but the lack of a fully $(S, \Phi)$-symmetric infinitesimal flex means that the orbit matrix is of full rank. So we are seeking new results and will need new techniques.

The fully symmetric Maxwell's Rule $(r=c-m)$ gives the standard necessary counts on $G, S$, and $\Phi$ for independence and full rank of an orbit matrix with $c$ columns, $r$ rows, and a space of trivial fully $(S, \Phi)$-symmetric infinitesimal motions (kernel of the orbit matrix for the complete graph) of dimension $m$. As usual, there are some added necessary conditions for independence of the rows which come from subgraphs $G^{\prime}$ of the graph $G$ :

1. If the rows of the orbit matrix are independent, then for each fully symmetric subgraph $G^{\prime}$ (generating $r^{\prime}$ rows and $c^{\prime}$ columns, as well as $m^{\prime}$ trivial infinitesimal motions for these columns), we have $r^{\prime} \leq c^{\prime}-m^{\prime}$;

2. If $H$ is a subgraph of $G$ such that $H$ and $x(H)$ share at most one vertex for each $x \in S$, then $|E(H)| \leq d|V(H)|-\left(\begin{array}{c}d+1 \\ 2\end{array}\right)$, where the framework is in dimension $d$, with $|V(H)| \geq d$.

Notice that we do not add special conditions for 'small' subgraphs in part 1 above. The reference to $m^{\prime}$ actually codes for all those special cases.

How could we generate sufficient conditions? One traditional way for the standard rigidity matrix has been to start with minimal examples, and use inductive techniques which preserve the independence and full rank of the rigidity matrix. These techniques include versions of vertex addition, edge splitting, and vertex splitting. This has been extended to fully symmetric inductive techniques, still with the standard rigidity matrix, in $[32,36]$. Transferred to the orbit matrix, such fully symmetric techniques will still preserve the independence and the full rank of the orbit matrix. However, there are many more inductive techniques which preserve the full rank of the orbit matrix - but would not preserve the full rank of the original rigidity matrix, since they would leave infinitesimal flexes which are not fully symmetric. For example, simply adding a vertex along the axis of a 2-fold rotation in 3-space (which adds one column) will only require one added edge orbit-which could be one edge (along the axis) or two edges (the orbit of a single edge) and this would definitely not generate an infinitesimally rigid framework in 3-space!

For 3-fold rotational symmetry in the plane, the symmetric inductive techniques in [36] do provide a full characterization for the corresponding orbit matrix to be independent and of full rank. However, it is unclear whether there are other symmetry groups for which the full characterization is accessible. When we find such a characterization, we will have a fully symmetrized version of the pebble game, for the orbit multi-graph.

\subsection{Geometric Conditions for Lower Rank in the Orbit Matrix}

For standard rigidity, there has been an algebraic geometric exploration of when a specific configuration $p$ makes a generically rigid graph $G$ into an infinitesimally 


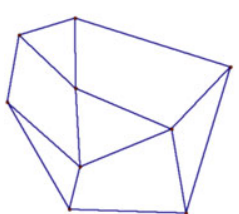

(a)

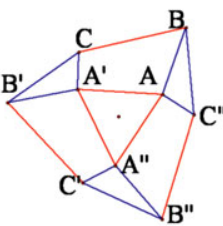

(b)

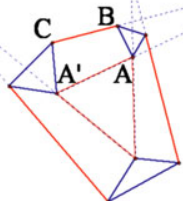

(c)

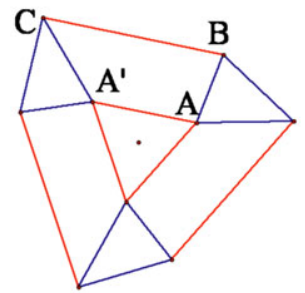

(d)

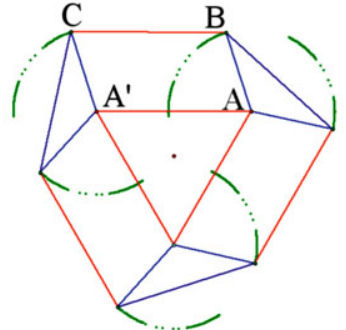

(e)

Fig. 10 The graph in (a) is generically rigid, and is also symmetry generically rigid for 3-fold rotation (b). The geometric condition for a non-trivial infinitesimal motion is three collinear induced points (c), which can also be achieved with 3-fold symmetry (d). Only the configuration (e), with parallelograms $A B C A^{\prime}$ have symmetry-preserving finite flexes

flexible framework $(G, p)$. The conditions are expressed in terms of a polynomial pure condition in the coordinates of $p$ which is $=0$ if and only if $(G, p)$ is infinitesimally flexible [43]. There will be a comparable theory for when configurations lower the symmetry generic rank of the orbit matrix. We illustrate the layers of this for a specific plane example with $C_{3}$ symmetry.

Example 9.1 Consider the framework illustrated in Fig. 10(a). The graph is generically rigid, and the pure condition for a lower rank of the rigidity matrix can be simplified to: make any of the four triangles collinear or make the induced points in Fig. 10(c) collinear.

Symmetry generic realizations with $\mathcal{C}_{3}$ symmetry are still infinitesimally rigid (Fig. 10(b)). Assuming $\mathcal{C}_{3}$ symmetry, the condition for an infinitesimal flex is that the three collinear points lie at infinity-or equivalently that the pairs of lines $A A^{\prime}, B C$ are parallel (Fig. 10(d)).

This is not enough for a fully symmetric infinitesimal flex-or equivalently for a drop in the rank of the orbit matrix. A direct geometric analysis verifies that the geometric condition for a fully symmetric infinitesimal flex (i.e. a drop in the rank of the orbit matrix) is that the three congruent faces $A, B, C, A^{\prime}$ are parallelograms (Fig. 10(e)). From the geometric theory of such structures of parallelograms and triangles, it is known that this infinitesimal flex is a finite, symmetry-preserving flex. Thus, we can express the condition on a configuration lowering the rank of the orbit matrix in terms of a polynomial in the representative vertices $A, B, C$ and image of $A$ under $C_{3}$. 
This example suggests that there is some interesting algebraic geometry to explore here.

\subsection{Transfer to Other Metrics}

The paper [31] presents results about the transfer of first-order rigidity properties (essentially all properties of the rigidity matrix) among frameworks which realize a given graph, on the same projective configuration, in the metric spaces $\mathbb{E}^{d}, \mathbb{S}^{d}$ and $\mathbb{H}^{d}$. What about a transfer of the orbit matrix for a symmetry group in $\mathbb{E}^{d}$ to the other metrics with the same symmetry groups?

In $\mathbb{E}^{d}$, all groups of isometries for a framework are point groups (there is a fixed point). These other spaces also share these same point groups-a connection that can be seen by coning up a dimension and then slicing the cone along a corresponding unit sphere. $\mathbb{S}^{d}$ and $\mathbb{H}^{d}$ have additional groups of isometries which do not fix a point and these can vary from space to space.

For simplicity, consider a point group in $\mathbb{E}^{d}$ and a sphere $\mathbb{S}^{d}$ tangent to the Euclidean space at the central point of the group. It is not hard to give a correspondence to a point group in the spherical space as well as a correspondence between symmetry generic frameworks in the two spaces. This correspondence will conserve fully symmetric infinitesimal flexes, fully symmetric trivial infinitesimal motions, and fully symmetric self-stresses. In short, the orbit matrices of the two configurations in the two metrics will have a simple invertible correspondence generated by multiplication on the right and left by appropriate invertible matrices [37].

Underlying this transfer is the operation of symmetric coning-with a new vertex in the next dimension, which is on the normal to the lower dimension and extends the axes and mirrors in the lower space in a way that conserves the group, and preserves symmetry, including finite flexes.

A similar process transfers orbit matrices and the predictions of finite flexes among $\mathbb{E}^{d}, \mathbb{S}^{d}$, and $\mathbb{H}^{d}$. This transfer gives a simple derivation of prior results on the flexibility of classes of Bricard octahedra and cross-polytopes in the spherical and hyperbolic metrics [1]. It is unusual for flexibility to transfer-so symmetry is a special situation. This transfer extends to other spaces with the same underlying projective geometry, such as the Minkowskian metric, provided that the point group is also realized as isometries in this metric. The full exploration of this transfer is the topic of continuing exploration, and further details and results will be presented in [37].

These other spaces such as $\mathbb{S}^{d}$ have additional symmetry groups which are not point groups (do not fix any point, or pair of antipodal points) and hence do not correspond to the symmetries in $\mathbb{E}^{d}$. There will be orbit matrices for these groups as well, and hence we can study these cases using a direct extension of the methods presented in this paper. These connections will be further explored in [37].

\subsection{Extensions to Body-Bar Frameworks}

One now standard extension of bar and joint frameworks are the body-bar frameworks $[15,44]$. These are a special class of frameworks, which in dimensions 3 and higher have a complete characterization for the multi-graphs which are generically isostatic 
(rigid, independent). The basic analysis of symmetry adapted rigidity matrices for these structures has been presented in [21].

It is clear that there are corresponding orbit matrices for body-bar frameworks, since they have bar and joint models, and the desired orbit matrix can, in principle, be extracted from that. The counting of columns and rows can also be adapted-though it would be helpful to give this in full detail.

A further extension studies body-hinge frameworks, with an emphasis on molecular models, where bodies (atoms) are connected by bonds (sets of 5 bars). The molecular models also have bar and joint models, so in principle there are corresponding orbit matrices, and counts to predict finite flexes. The classical 'boat and chair' configurations of cyclohexane in chemistry (a ring of six carbons) is an example where 3 -fold symmetry (the chair) keeps the generic first-order rigidity and independence, and the 2-fold symmetry (the boat) is a model of the flexible octahedron.

Theorem 7.5 showing the flexibility of generically isostatic graphs in 3-space realized with 2-fold symmetry, extends from this example to general molecules in 3-space with 2-fold symmetry and no atoms or bonds intersecting the axis. This is a common occurrence among dimers of proteins, so it has potential applications to the study of proteins [50].

\subsection{Orbit Matrices for Other Geometric Constraint Systems}

Owen and Power have investigated other examples of geometric constraints in CAD under symmetry [26] (see also [38, 48]). In general, constraint systems with matrix representations are open to analysis using group representations and symmetric block decompositions of their matrices. However, there are some surprises which confirm that the analysis of corresponding orbit matrices may not be a simple translation of the results given here.

It is well known that in the plane, infinitesimal motions correspond to parallel drawings of the same geometric graph and configuration. The correspondence involves turning all the velocities by $90^{\circ}$, which takes a trivial rotation to a trivial dilation. For symmetry, this turn takes an infinitesimal motion which is fully symmetric for a rotation to a parallel drawing which is fully symmetric for the same rotation. So our techniques transfer immediately to parallel drawings of frameworks with purely rotational point groups. However, the operation of turning the velocities by 90 degrees takes an infinitesimal motion which is fully symmetric for a mirror to a parallel drawing which is anti-symmetric for the same mirror (and vice versa). This clearly

Fig. 11 For frameworks with mirror symmetry the space of fully symmetric trivial parallel drawings may be larger than the space of fully symmetric trivial infinitesimal motions (a), and frameworks without fully symmetric infinitesimal flexes may have fully symmetric parallel drawings (b)

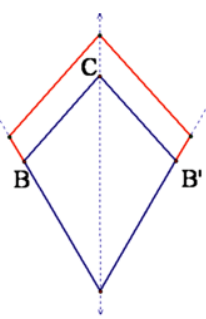

(a)

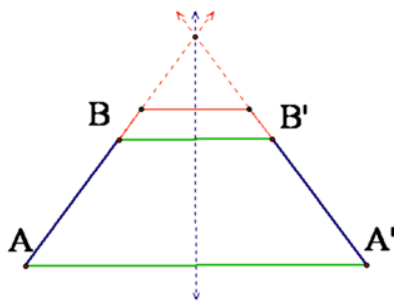

(b) 
leads to changes in the development of the orbit matrix. There are also additional fully symmetric trivial motions (dilations about the center of the point group are trivial, for the mirror). More surprisingly, some edge orbits seem to disappear in the obit matrix (edge $A A^{\prime}$ in Fig. 11(b)). Figure 11 illustrates two examples. This may be enough to confirm that the extensions to other constraint systems are non-trivial, and worth carrying out!

\section{References}

1. Alexandrov, V.: Flexible polyhedra in the Minkowski 3-space. Manuscr. Math. 11(3), 341-356 (2003)

2. Asimov, L., Roth, B.: The rigidity of graphs. Trans. Am. Math. Soc. 245, 279-289 (1978)

3. Baker, E.: An analysis of the bricard linkages. Mech. Mach. Theory 15, 267-286 (1980)

4. Bishop, D.M.: Group Theory and Chemistry. Clarendon Press, Oxford (1973)

5. Bolker, E.D., Roth, B.: When is a bipartite graph a rigid framework? Pac. J. Math. 90, $27-44$ (1980)

6. Borcea, C.S., Streinu, I.: Periodic frameworks and flexibility. Proc. R. Soc., Math. Phys. Eng. Sci. 466, 2633-2649 (2010)

7. Bottema, O.: Die Bahnkurven eines merkwürdigen Zwölfstabgetriebes. Österr. Ing.-Arch. 14, 218222 (1960)

8. Bricard, R.: Mémoire sur la théorie de l'octaèdre articulé. J. Math. Pures Appl. 5(3), 113-148 (1897)

9. Cauchy, A.L.: Sur les polygons et les polyèdres. Oevres Complètes d'Augustin Cauchy 2è Série Tom 1, 26-38 (1905)

10. Connelly, R.: A counterexample to the rigidity conjecture for polyhedra. Inst. Haut. Etud. Sci. Publ. Math. 47, 333-335 (1978)

11. Connelly, R.: Highly symmetric tensegrity structures. http://www.math.cornell.edu/ tens/ (2008)

12. Connelly, R.: The rigidity of suspensions. J. Differ. Geom. 13(3), 399-408 (1978)

13. Connelly, R.: Rigidity and energy. Invent. Math. 66, 11-33 (1982)

14. Connelly, R., Fowler, P.W., Guest, S.D., Schulze, B., Whiteley, W.: When is a symmetric pin-jointed framework isostatic? Int. J. Solids Struct. 46, 762-773 (2009)

15. Connelly, R., Jordán, T., Whiteley, W.: Generic global rigidity of body-bar frameworks. Egerváry Research Group on Combinatorial Optimization. Technical Report TR-2009-13 (2009)

16. Crapo, H., Whiteley, W.: Statics of frameworks and motions of panel structures, a projective geometric introduction. Topol. Struct. 6, 43-82 (1982)

17. Crapo, H., Whiteley, W.: Spaces of stresses, projections, and parallel drawings for spherical polyhedra. Contrib. Algebra Geom./Beitrage Algebra Geom. 35, 259-281 (1994)

18. Fowler, P.W., Guest, S.D.: A symmetry extension of Maxwell's rule for rigidity of frames. Int. J. Solids Struct. 37, 1793-1804 (2000)

19. Graver, J.E., Servatius, B., Servatius, H.: Combinatorial Rigidity. Graduate Studies in Mathematics. AMS, Providence (1993)

20. Guest, S.D., Fowler, P.W.: Symmetry conditions and finite mechanisms. Mech. Mater. Struct. 2(6), 293-301 (2007)

21. Guest, S.D., Schulze, B., Whiteley, W.: When is a symmetric body-bar structure isostatic? Int. J. Solids Struct. 47, 2745-2754 (2010)

22. Hall, L.H.: Group Theory and Symmetry in Chemistry. McGraw-Hill, New York (1969)

23. Laman, G.: On graphs and rigidity of plane skeletal structures. J. Eng. Math. 4, 331-340 (1970)

24. Kangwai, R.D., Guest, S.D.: Detection of finite mechanisms in symmetric structures. Int. J. Solids Struct. 36, 5507-5527 (1999)

25. Kangwai, R.D., Guest, S.D.: Symmetry-adapted equilibrium matrices. Int. J. Solids Struct. 37, 15251548 (2000)

26. Owen, J.C., Power, S.C.: Frameworks, symmetry and rigidity. Preprint (2009)

27. Malestein, J., Theran, L.: Generic combinatorial rigidity of periodic frameworks. Preprint, arXiv:1008.1837 (2010)

28. Ross, E.: Combinatorial and geometric rigidity of periodic structures. Draft Ph.D. thesis, York University, Toronto, ON, Canada. To appear

29. Ross, E., Schulze, B., Whiteley, W.: Finite motions from periodic frameworks with added symmetry. Preprint, arXiv:1010.5440 (2010) 
30. Roth, B., Whiteley, W.: Tensegrity frameworks, Amer. Math. Soc. 266(2), 419-446 (1981)

31. Saliola, F.V., Whiteley, W.: Some notes on the equivalence of first-order rigidity in various geometries. arXiv:0709.3354 (2007)

32. Schulze, B.: Combinatorial and geometric rigidity with symmetry constraints. Ph.D. thesis, York University, Toronto, ON, Canada (2009)

33. Schulze, B.: Symmetry as a sufficient condition for a finite flex. SIAM J. Discrete Math. 24(4), 1291$1312(2010)$

34. Schulze, B.: Block-diagonalized rigidity matrices of symmetric frameworks and applications. Beitrage Algebra Geom. 51(2), 427-466 (2010)

35. Schulze, B.: Injective and non-injective realizations with symmetry. Contrib. Discret. Math. 5(1), 59$89(2010)$

36. Schulze, B.: Symmetric versions of Laman's Theorem. Discrete Comput. Geom. 44(4), 946-972 (2010)

37. Schulze, B., Whiteley, W.: Coning, symmetry, and spherical frameworks (2010, in preparation)

38. Servatius, B., Whiteley, W.: Constraining plane configurations in CAD: combinatorics of directions and lengths. SIAM J. Algebr. Discrete Methods 12, 136-153 (1999)

39. Stachel, H.: Zur Einzigkeit der Bricardschen Oktaeder. J. Geom. 28, 41-56 (1987)

40. Stachel, H.: Flexible cross-polytopes in the Euclidean 4-space. J. Geom. Graph. 4(2), 159-167 (2000)

41. Stachel, H.: Flexible octahedra in the hyperbolic space. Math. Appl. (János Bolyai memorial volume) 581, 209-225 (2006)

42. Tarnai, T.: Finite mechanisms and the timber octagon of Ely cathedral. Topol. Struct. 14, 9-20 (1988)

43. White, N., Whiteley, W.: The algebraic geometry of stresses in frameworks. SIAM J. Algebr. Discrete Methods 4, 481-511 (1983)

44. White, N., Whiteley, W.: The algebraic geometry of bar and body frameworks. SIAM J. Algebr. Discrete Methods 8, 1-32 (1987)

45. Whiteley, W.: Cones, infinity and one-story buildings. Topol. Struct. 8, 53-70 (1983)

46. Whiteley, W.: Infinitesimally rigid polyhedra I. Statics of frameworks. Trans. Am. Math. Soc. 285(2), 431-465 (1984)

47. Whiteley, W.: Infinitesimal motions of a bipartite framework. Pac. J. Math. 110, 233-255 (1984)

48. Whiteley, W.: A matroid on hypergraphs, with applications in scene analysis and geometry. Discrete Comput. Geom. 4, 75-95 (1989)

49. Whiteley, W.: Some matroids from discrete applied geometry. Contemp. Math. 197, 171-311 (1996)

50. Whiteley, W.: Counting out to the flexibility of molecules. Phys. Biol. 2, 1-11 (2005) 